\title{
Enormous Cloud Cover on Venus observed by Akatsuki's IR2 data recovered by Restoration-by- Deconvolution (RD) method
}

Choon Wei Vun ( $\sim$ anthonyvun@hotmail.com )

Q

https://orcid.org/0000-0003-

4449-0737

\section{Takehiko Satoh}

Institute of Space and Astronautical Science (ISAS/JAXA)

Takao M. Sato

Hokkaido Information University

Takeshi Horinouchi

Hokkaido University

Full paper

Keywords: Venus, Akatsuki, Atmosphere, Aerosols, Near-Infrared, Image Processing

Posted Date: May 11th, 2020

DOI: https://doi.org/10.21203/rs.3.rs-26756/v1

License: (9) This work is licensed under a Creative Commons Attribution 4.0 International License.

Read Full License 


\section{Title: Enormous Cloud Cover on Venus observed by Akatsuki's}

\section{IR2 data recovered by Restoration-by-Deconvolution (RD) method}

3

4 Author 1: Choon Wei Vun, Department of Space and Astronautical Science, The Graduate

5 University for Advanced Studies, SOKENDAI, 3-1-1 Yoshinodai, Chuo-ku, Sagamihara,

6 Kanagawa 252-5210, Japan, anthonyvun@hotmail.com

7

8 Author 2: Takehiko Satoh, Institute of Space and Astronautical Science, Japan Aerospace

9 Exploration Agency, 3-1-1 Yoshinodai, Chuo-ku Sagamihara, Kanagawa 252-5210, Japan,

10 satoh@stp.isas.jaxa.jp

11

12 Author 3: Takao M. Sato, Space Information Center, Hokkaido Information University, 59-2,

13 Nishinopporo, Ebetsu, Hokkaido 069-8585, Japan, sato.takao@do-johodai.ac.jp

14

15 Author 4: Takeshi Horinouchi, Faculty of Environmental Earth Science, Hokkaido University,

16 Nishi 5 Kita, Kita 10, Kita-ku, Sapporo, Hokkaido 060-0810, Japan,

17 horinout@ees.hokudai.ac.jp

18 


\section{Abstract}

20 We have developed a new method 'Restoration by Deconvolution' (RD) to restore nightside 21 photometry in the Akatsuki/IR2 images contaminated by spread light from intense dayside crescent. With updated point spread function model for IR2 and incorporation of radiative transfer computations, our RD-method is able to improve the photometric accuracy of nightside emission data in both $2.26 \mu \mathrm{m}$ and $1.735 \mu \mathrm{m}$ filters. Exploiting the enhanced photometric quality, the 'Enormous Cloud Cover' (ECC) features observed in both 2016-08-18 and 201608-27 data have been investigated. Possible altitude variations in the ECC's spatial variation measurements from $\mathrm{z}=52 \mathrm{~km}$ up to $\mathrm{z}=60 \mathrm{~km}$ were found. The observations were interpreted that ECC constituting of large sulfuric acid droplets (mean radius of $3.65 \mu \mathrm{m}$ and the optical thickness 7-9) experience strong upwelling near the ECC front. This elevates the aerosols up to $\mathrm{z}=58-60 \mathrm{~km}$, then subjected upon by strong downwelling forces the particles to sink by a velocity $\sim-0.3 \mathrm{~m} / \mathrm{s}$. The analyses of physical properties and evolutionary behavior of the ECC on both dates (08-18 and 08-27) suggest them to be recurring phenomena in the lower part of Venus' clouds.

\section{Keywords}

Venus, Akatsuki, Atmosphere, Aerosols, Near-Infrared, Image Processing

\section{Introduction}

39 Venus is shrouded by thick clouds of which structures have been explored by various measurements: multiwavelength observations (imaging and spectroscopy), in-situ probe and

41 landers, and radio occultation. The clouds have significant influence in the energy budget, 42 meteorology, microphysical and chemical processes in the atmosphere. Past explorations by 
43 Pioneer Venus (PV), Venera and Vega entry probes (Esposito et al, 1983) have discovered the

44 cloud's main deck situating in between $\sim 47$ to $70 \mathrm{~km}$ altitudes is vertically stratified into three distinctive layers (upper, middle, and lower clouds) accompanied by tenuous hazes above and below the cloud deck. The upper clouds observed by reflected sunlight are opaque and featureless and exhibiting contrasts when observed in the ultraviolet regime (Pollack et al., 1979). On the other hand, the middle and lower clouds $(47.5-56.5 \mathrm{~km}$ altitudes $)$ can be probed by infrared remote sensing to observe both dayside (Belton et al., 1991; Peralta et al., 2018) and nightside (Allen \& Crawford, 1984). The lower clouds are constantly being heated from below by thermal radiation originating from the hot lower atmosphere and the ground. This drives convection in the lower cloud layer (Imamura et al.2014, Baker et al., 1998) that activates the microphysical processes of the cloud particles to go through condensation, coagulation, and evaporation in this dynamical range (McGouldrick., 2017). Hence, the lower clouds play the key role in contributing to the Venusian greenhouse effect, local radiative energy balance, and cloud dynamics (Titov et al., 2018; McGouldrick et al., 2012).

Based on PV's Cloud Particle Size Spectrometer (LCPS) studies, measuring particle sizes upon descent have evidenced the multimodal particle size distribution in the vertically stratified main cloud layers (Knollenberg and Hunten, 1980). Particle distribution mode $3(\overline{\mathrm{r}}=3.65 \mu \mathrm{m})$ was mainly found in the lower cloud layer. On the other hand, the mode $2(\overline{\mathrm{r}}=1.0 \mu \mathrm{m})$ and mode 2' $(\overline{\mathrm{r}}=1.4 \mu \mathrm{m})$ number densities dispersing throughout the main cloud deck, peaking in the lower clouds but in slightly different altitude regions (Ragent et al., 1985). The diffusional growth of mode 2 and mode 2' particles from sulfuric acid vapors can be transported downwards into the lower part of the cloud deck by eddy diffusion (Imamura and Hashimoto., 1998; McGouldrick., 2017). The lower clouds can be probed using the $\mathrm{CO}_{2}$ atmospheric window in near-infrared (NIR) regime (Allen \& Crawford, 1984). Akatsuki's IR2 camera has 

three filters $(1.735 \mu \mathrm{m}, 2.26 \mu \mathrm{m}$, and $2.32 \mu \mathrm{m})$ to observe the lower clouds in Venus's nightside (Satoh et al., 2016). Contrast features emerging in these filters are due to spatially inhomogeneous scattering and absorption of the radiation from the lower atmosphere by cloud particles. Until now, the morphology studies have been performed by using image enhancing techniques to identify interesting features (Peralta et al., 2019, Horinouchi et al., 2017). However, accurate photometric studies have never been performed due to data contamination by dayside light until the development of imagery restoration methods: 'Restoration by Simple Subtraction' (Satoh et al., submitted) and 'Restoration by Deconvolution' (this paper). Also, the aerosol properties can be investigated utilizing different filters in IR2 $(2.26 \mu \mathrm{m}$ and $1.735 \mu \mathrm{m})$ such as analyses done on observations by Galileo Near Infrared Mapping Spectrometer (NIMS) during Venus flyby and Visible and Infrared Thermal Imaging Spectrometer (VIRTIS) on board Venus Express (VEx) to evaluate aerosol size distributions using $2.30 \mu \mathrm{m}$ and $1.74 \mu \mathrm{m}$ infrared radiations (Carlson et al., 1993; Wilson et al., 2008).

Venus is dominated by a global atmospheric circulation known as the super-rotation (Schubert, 1983). In contrast to the slow rotating planet body (1.6 m/s westward), the wind speed can reach up to $\sim 100 \mathrm{~m} / \mathrm{s}$ near the cloud top ( $70 \mathrm{~km}$ altitude) whereas it slows down to $\sim 70 \mathrm{~m} / \mathrm{s}$ in the lower clouds $\mathrm{z} \sim 50 \mathrm{~km}$ (Carlson et al., 1991; Hueso et al., 2012). In the observations by Akatsuki, there have been reports of the occurrence of 'dark-markings' or 'discontinuity' features (Peralta et al., submitted). These 'discontinuity' features can be described as a sharp change in the cloud opacity that results in distinct boundaries in observed transmission of clouds propagating westwards. Peralta et al. (submitted) suggested that the 'discontinuity' features can propagate at speeds of $\sim 90 \mathrm{~m} / \mathrm{s}$. Amongst these findings, two events on Aug-18 and Aug-27 were the largest discontinuity features observed by Akatsuki are also termed as

92 'Enormous Cloud Cover' (ECC) in this paper. At present, there have been dynamical studies 
93 suggesting mechanisms making the ECC feature could be manifestation of Kelvin waves

94 (Peralta et al., submitted). However, the actual compositions of aerosols were not well known due to the light contamination over the IR2 nightside data. This becomes the main motivation

96 for the purpose of this paper to supply accurate photometric evidences utilizing enhanced RD 97 processed images.

98

99 Satoh et al., (submitted) has improved data qualities of Akatsuki/IR2 by developing the

100 'Restoration by Simple Subtraction' (RSS) method which separates the nightside emission

101 features from the strong contamination due to spread of dayside light. This has enabled quality

102 photometric studies for the first time ever. However, the RSS method is unable to restore light

103 spreading property of the nightside photometry by the IR2 camera's PSF. This leads to the

104 scope of this paper to further improve the photometric accuracy by true contrast level using deconvolution technique in 'Restoration by Deconvolution' (RD) method.

107 The main outline of this paper would begin with data selection for restoration targets and 108 photometric analyses (Section 2), introducing the development of RD method (Section 3), 109 evaluating aerosol properties of the ECC (Section 4), and finally to discuss the possible 110 interpretations of the ECC's microphysical properties (Section 5).

\section{2. Data}

\section{2.1. IR2 data}

114 The IR2 camera onboard Akatsuki utilizes narrow band filters in the atmospheric windows to

115 probe through the middle and lower clouds of Venus atmospheric windows (Satoh et al., 2016).

116 The three filters of the IR2 camera used to observe the nightside are $1.735 \mu \mathrm{m}, 2.26 \mu \mathrm{m}$, and $1172.32 \mu \mathrm{m}$. Infrared radiation originating from lower altitudes are attenuated by $\mathrm{CO}_{2}$ absorption 
in $1.735 \mu \mathrm{m}$ and $2.26 \mu \mathrm{m}$, whilst $2.32 \mu \mathrm{m}$ contains $\mathrm{CO}$ absorption band. To save downlink time

119 from Akatuski, 'Region of Interest' (ROI) function of the onboard computer trims the image

120 frame of 1024 x 1024 pixels (thereinafter denoted as ' $1 \mathrm{k}$-frame') into smaller pixel frame size

121 [X1:X2, Y1:Y2] focusing only on region for Venus disk observations. The IR2 camera is

122 operated under cooling temperature of the platinum silicide (PtSi) detector regulated by the

123 cryocooler to be below $70 \mathrm{~K}$. This is crucial to Venus nightside observations because the

124 thermally induced electrons saturate the detector within few tens of seconds when the detector

125 temperature is 70K (Satoh et al., 2016).

127 The data used in this paper (Table $1 \mathrm{a}$ and $1 \mathrm{~b}$ for $2.26 \mu \mathrm{m}$ and $1.735 \mu \mathrm{m}$ filters respectively) are

128 L2B data available from "Akatsuki 2- $\mu$ m Camera (IR2) Data Archive" on Data Archives and

129 Transmission System (DARTS) (Murakami et al., 2018). L2B data are observations by IR2

130 camera in $1.735 \mu \mathrm{m}, 2.26 \mu \mathrm{m}$, and $2.32 \mu \mathrm{m}$ filters. L2B data for the three filters experience data

131 contamination by the camera's PSF and intense dayside crescent and pixel saturation when the

132 number of photoelectrons exceeds the upper limit of the pixel's potential well (Examples

133 shown in Figure 1). The overall output of L2B images appears to have a dependency on

134 operating temperature. This is described as temperature-dependent sensitivity of IR2 and the

135 data were corrected using functions described in Satoh et al. (submitted). The correction for

136 geographic mapping was conducted by improved image navigation maximizing the radial

137 (inward) component of the gradient of radiance (Horinouchi et al., 2017).

139 We have selected data from orbit 24 and 25 having wide spatial and temporal coverage on

140 Venus nightside observations. However, we focus especially on the data of dates Aug-18 (from

141 orbit 24) and Aug-27 (from orbit 25). The main reason to this is to focus observations on the

142 'Enormous Cloud Cover' (ECC) features. Data from these two dates during apoapsis passage 
143 observing Venus are ideal for investigating daylight spreading by point spread function (PSF)

144 while high resolution nightside observations are as frequent as 2-hours intervals. Note that L2B

145 images from these observations were trimmed by the ROI function i.e. [129:896, 385:1024].

\section{3. Methodology}

149 The condition for deconvolution process requires the saturated pixels being estimated in L2B

150 under all possible factors influencing the IR2 observations. Then, deconvolution by model-

151 PSF can be performed on the entire observing frame. Thus, the steps (Figure 2a) to preparing 152 for the Restoration-by-Deconvolution (RD) are: Step 1. Modelling Point Spread Function 153 (PSF); Step 2. Modeling dayside and nightside radiances using radiative transfer; Step 3.

154 'Model Venus Disk' (Dayside + Nightside) convolved by model-PSF; Step 4. Saturated pixels 155 in L2B replaced by 'Convolved Model' from (Step 3); Step 5. (Step 4) undergoes 156 Deconvolution by model-PSF. The sequential image preparations from Step 1 to Step 5 are 157 summarized in Figure 2b.

\subsection{Modelling the IR2 Point Spread Function (PSF)}

162 We modified the mathematical approximation introduced by Satoh et al. (2017) that initially

163 incorporates both point-symmetric and axisymmetric terms to include an additional diffusive 164 term. The current PSF reported in this paper also incorporates updated parameters for 165 approximating both the point-symmetric and axisymmetric terms. 
167 The three components of the PSF model are: a point-symmetric term $\left[a_{r}(r)\right]$ and two

168 axisymmetric terms $\left[\mathrm{a}_{\mathrm{X}}(\mathrm{X})\right.$ and $\left.\mathrm{a}_{\mathrm{Y}}(\mathrm{Y})\right]$ from Satoh et al. 2017; and a newly introduced 169 diffusive term $\mathrm{d}(\mathrm{X}, \mathrm{Y})$.

$$
\operatorname{PSF}(X, Y)=g_{1} a_{r}(r)+g_{2} a_{X}(X) \times a_{Y}(Y)+d(X, Y)
$$

170 where

$$
r^{2}=X^{2}+Y^{2}
$$

$$
\mathrm{X}=\mathrm{x}-\mathrm{x}_{0}
$$

$$
\mathrm{Y}=\mathrm{y}-\mathrm{y}_{0}
$$

174 where $\left(\mathrm{x}_{0}, \mathrm{y}_{0}\right)$ is the center of the PSF.

175 The point symmetric term $\mathrm{a}_{\mathrm{r}}(\mathrm{r})$, and axisymmetric terms $\mathrm{a}_{\mathrm{X}}(\mathrm{X})$ and $\mathrm{a}_{\mathrm{Y}}(\mathrm{Y})$ are expressed as 176 the following (Satoh et al., 2017):

$$
\begin{aligned}
& \mathrm{a}_{\mathrm{r}}(\mathrm{r})=\frac{\Gamma_{1}}{2 \pi\left[\mathrm{r}^{2}+\left(\Gamma_{1} / 2\right)^{2}\right]^{\mathrm{e}_{1}}} \\
& \mathrm{a}_{\mathrm{X}}(\mathrm{X})=\frac{\Gamma_{2}}{2 \pi\left[\mathrm{X}^{2}+\left(\Gamma_{2} / 2\right)^{2}\right]^{\mathrm{e}_{2}}} \\
& \mathrm{a}_{Y}(\mathrm{Y})=\frac{\Gamma_{2}}{2 \pi\left[\mathrm{Y}^{2}+\left(\Gamma_{2} / 2\right)^{2}\right]^{\mathrm{e}_{2} \mathrm{c}}}
\end{aligned}
$$

177 where the parameters were updated as the following:

\begin{tabular}{llllll}
\hline$\Gamma_{1}$ & 0.30 & $\mathrm{e}_{1}$ & 1.18 & $\mathrm{~g} 1$ & 3.30 \\
$\Gamma_{2}$ & 8.0 & $\mathrm{e} 2$ & 0.83 & $\mathrm{c}$ & 1.30 \\
\hline
\end{tabular}

178

179 In the latest version of PSF modelling, the region within the first halation ring is a 'hole' in the

180 axisymmetric term. This is to simulate the perfect internal reflection during first point of contact of remnant light in the PtSi substrate. In addition, we incorporate another diffusive term 
$d(X, Y)$ to that spreads further away from the intensive light source. This may be due to light

183 reflections that results in out-focused 'ghosts' of the bright object.

184 The diffusive term $\mathrm{d}(\mathrm{X}, \mathrm{Y})$ is defined as follow:

$$
\mathrm{d}(\mathrm{X}, \mathrm{Y})=1.4 \times 10^{-6} \times \exp \left[-\left(\frac{\mathrm{r}}{400}\right)^{2}\right]
$$

185 The closeness of our model-PSF can be compared with the light spreading properties in L2B

186 data. We selected $2.02 \mu \mathrm{m} \mathrm{L2B}$ data from orbit 14,15 , and 16 (dayside observations) below

$18745^{\circ}$ phase angle to analyze the PSF properties. In each observation, the Venus disk is being 188 masked to measure the total amount of flux outside the disk (termed as 'Loss to off-disk flux').

189 The ratio of 'Loss to off-disk flux' to 'total flux' is being plotted against the spacecraft distance 190 from Venus (Figure 3). Similarly, we take same measurements for our model PSF-convolved 191 dayside (where optical fogging was also included). Light spreading properties by our model192 PSF were found to match well to those of the observations as illustrated in Figure 3.

\subsection{Radiative transfer calculation}

\subsubsection{Discrete ordinate radiative transfer for nightside emission}

198 The Radiative Transfer computation incorporates "discrete ordinate RT" developed by

199 Stamnes et al. (1988) combining with the line-by-line radiative transfer to calculate transmittance spectra for sub-layers of Venus atmosphere. The computation treats Venus atmospheres as stacked layers each of which is 2-km thick. Each layer was assumed to be homogeneous in temperature, pressure, and molecular and aerosol compositions. Then, the atmospheric Pressure-Temperature profile (2-km resolution) was obtained from observations by PV probe by Seiff (1982). The molecular lines implemented were obtained from 'Highresolution Transmission' (HITRAN) molecular absorption database for $\mathrm{CO}_{2}, \mathrm{~N}_{2}, \mathrm{CO}, \mathrm{H}_{2} \mathrm{O}$, and 
$\mathrm{HCl}$. Also, the radiative transfer calculation assumes the aerosols are droplets of $85 \% \mathrm{H}_{2} \mathrm{SO}_{4}$

207 aqueous solution (Palmer \& Williams, 1975), and the size-distribution parameters were extracted from Grinspoon et al. (1993). Whereas, absorptions by continuum were set as $5.6 \times 10^{-9} \mathrm{~cm} 1$ amagat2 at $1.735 \mu \mathrm{m}$ and $3.5 \times 10^{-8} \mathrm{~cm}_{1}$ amagat2 at $2.26 \mu \mathrm{m}$.

210

211 The nominal cloud model incorporates the particle mode distributions locating at different

212 altitudes within the middle and lower clouds where absorptions above $62 \mathrm{~km}$ were neglected:

$213 \mathrm{z}=48-52 \mathrm{~km}$ (mode 3, $\tau=14) ; \mathrm{z}=52-54 \mathrm{~km}$ (mode 2', $\tau=14) ; \mathrm{z}=56-60 \mathrm{~km}$ (mode 2, $\tau=5) ; \mathrm{z}=60$ -

$21462 \mathrm{~km}$ (mode $1, \tau=2$ ). This trimodal distribution is summarised in Table $3 \mathrm{a}$ and extinction cross

215 sections are summarised in Table $3 b$. The limb darkening equations attained from the radiative 216 transfer are presented as follow (Satoh et al., submitted):

$$
\mathrm{I}_{1.735 \mu \mathrm{m}}^{\prime}=\frac{\mathrm{I}_{1.735 \mu \mathrm{m}}}{0.6423 \cos \left(\theta_{\mathrm{em}}\right)+0.3577}
$$

$$
\mathrm{I}_{2.26 \mu \mathrm{m}}^{\prime}=\frac{\mathrm{I}_{2.26 \mu \mathrm{m}}}{0.6904 \cos \left(\theta_{\mathrm{em}}\right)+0.3096}
$$

219 where $\mathrm{I}^{\prime}$ is limb-darkening corrected intensity, I is observed intensity, and $\theta_{\mathrm{em}}$ is emission 220 angle.

\subsubsection{Preparation for 'Model Dayside' (MDD)}

The calculation for dayside reflectance was referred to the line-by-line radiative transfer by Sato et al. (2020) developed for modeling $2.02 \mu \mathrm{m}$ dayside intensities. This model was translated for calculating dayside intensities in both $2.26 \mu \mathrm{m}$ and $1.735 \mu \mathrm{m}$ filters to generate 


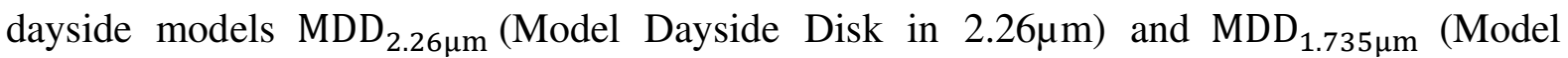

229 Dayside Disk in $1.735 \mu \mathrm{m})$. The dayside photometry calculated by radiative transfer accounts

230 for the nominal cloud model from Haus et al. (2015). Considering possible intrinsic changes

231 of spatial and temporal variations of Venus clouds from one image to another, we have to introduce a factor so-called 'Dayside F factor' (denoted as F) to adjust the dayside intensities based on observation conditions of every image (Appendix A).

\subsection{Preparation for 'Cleaned Nightside' (CLN)}

236 For 2.26 $\mu \mathrm{m}$, 'Cleaned Nightside' $\mathrm{CLN}_{2.26 \mu \mathrm{m}}$ was adapted from RSS-processed images (Satoh 237 et al., submitted). After acquiring 'Restoration-by-Deconvolution Data' $\left(\mathrm{RDD}_{2.26 \mu \mathrm{m}}\right)$, $238 \mathrm{RDD}_{2.26 \mu \mathrm{m}}$ can be used as a baseline to model $\mathrm{CLN}_{1.735 \mu \mathrm{m}}$. Both $2.26 \mu \mathrm{m}$ and $1.735 \mu \mathrm{m}$ being 239 in the windows of the $\mathrm{CO}_{2}$ atmosphere have slightly different contrast and photometric 240 emissions due to the differences in scattering properties by aerosols. $\mathrm{CLN}_{1.735 \mu \mathrm{m}}$ can be 241 estimated from $\mathrm{RDD}_{2.26 \mu \mathrm{m}}$ based on the conversion relationship described by emission 242 properties in the two filters $(1.735 \mu \mathrm{m}$ and $2.26 \mu \mathrm{m})$ as calculated by radiative transfer in 243 Equation 8 (Satoh et al., submitted)

$$
\log \left(\operatorname{CLN}_{1.735 \mu \mathrm{m}}\right)=\mathrm{m} \times \log \left(\mathrm{RRD}_{2.26 \mu \mathrm{m}}\right)-0.157
$$

244 where $\mathrm{m}=0.598$ is the conversion factor estimated by radiative transfer.

\subsection{Preparation for Deconvolution by PSF in RD-method: RDD}

247 As the observations suffer saturation when the capacity of each pixel is overflowed, both 248 photometry in saturated region and outside the ROI frame were lost. Thus, we have to model 249 the loss photometry to prepare the data for deconvolution process. 
251 Deconvolution requires all light spreading as much as possible to 're-absorb' the photometry extended by the long PSF tail back to its light source. Hence, we prepare a larger 2048 x 2048 pixels2 size canvas (denoted as '2k-frame') to accommodate model photometry estimation outside the ROI frame. The data used in this paper for Aug-18 and Aug-27 were trimmed by [129:896, 385:1024] in the ROI function. The observing CCD's 1k-frame is fixed into the middle of the prepared 2k-frame where light spreading by PSF outside the ROI frame can be accommodated. The use of larger canvas can also suppress repetition of artifacts when inverse Fourier transform of the entire frame is being applied in deconvolution.

'Model Dayside Disk' (MDD) (from Section 3.2.2) and 'Cleaned Nightside' (CLN) (from Section 3.3) together make up the full 'Model Day + Nightside' (MDN). The MDN can then be convolved by model-PSF giving 'Convolved Model' (CVM) where saturated pixels in L2B and the canvas vessel outside the ROI frame ( $2 \mathrm{k}$-frame) can be replaced to estimate loss photometry (Figure 4-right).

In this process, only saturated pixels were being replaced making up the 'Combined-image' (COM) while keeping unsaturated pixels unchanged. Now, this suffices the condition for RDmethod requiring the saturated pixels to be appropriately determined where deconvolution by model-PSF can be performed on the entire frame (COM).

\subsection{Calibration for optical Fogging}

274 In typical L2B observations, there are 'ghost-features' due to light remnants bouncing off the glass surfaces in the IR2 camera optics. Thus, this gives an overall offset photometry to the 
nightside observations. This offset radiance (also termed as 'fogging') is hence dependent on

277 the total incoming flux in which is also dependent on both phase angle and apparent diameter.

278 The fogging amount is summarized in Table 4 where they do not have significant influence in 279 both $2.26 \mu \mathrm{m}$ and $1.735 \mu \mathrm{m}$ filters. Refer to Figure 7 and 8 showing the upper limit of fogging 280 influence when fogging amount is multiplied by 10. The original L2B unsaturated pixels can 281 be subtracted by fogging value for each observation (Table 4) before the replacement for saturated pixels (giving COM) to suppress this minimal offset by fogging: L2B' $=$ L2B - Fog where L2B' is corrected L2B, and Fog is 'Fogging value' obtained from Table 4.

[Table 4]

\section{Results}

\subsection{Restoration by Deconvolution (RD) with Richardson-Lucy Algorithm}

The deconvolution incorporated in the RD-method utilizes the Richardson-Lucy (R-L) Algorithm (Lucy., 1974) which is an iterative computation that restores underlying pixels that has been contaminated by the PSF. Assuming we have an observed image that is expressed as:

$$
I(X, Y)=\int_{-\infty}^{\infty}\left[I_{o}(X, Y) \times \operatorname{PSF}(X, Y) d X d Y\right]+\delta(X, Y)
$$

where $\mathrm{I}(\mathrm{X}, \mathrm{Y})$ is the observed image, $\mathrm{I} 0(\mathrm{X}, \mathrm{Y})$ is the ideal image, $\mathrm{PSF}(\mathrm{X}, \mathrm{Y})$ is our model-PSF

294 (from Equation 1), and $\delta(\mathrm{X}, \mathrm{Y})$ is random noise associated to the observed image. Equation 9

295 can also be simplified as:

$$
\mathrm{I}(\mathrm{X}, \mathrm{Y})=\mathrm{I}_{0}(\mathrm{X}, \mathrm{Y}) * \mathrm{PSF}(\mathrm{X}, \mathrm{Y})+\delta(\mathrm{X}, \mathrm{Y})
$$

296 where ' $*$ ' means convolution in the Fourier transform space.

297 The R-L deconvolution algorithm can then be expressed as the following: 


$$
I_{n+1}(X, Y)=I_{n}(X, Y)\left(\frac{I_{0}(X, Y)}{I_{n}(X, Y) * P S F(X, Y)}\right) * \operatorname{PSF}^{\prime}\left(X^{\prime}, Y^{\prime}\right)
$$

299 where $n$ is number of iterations, $I_{n+1}(X, Y)$ is output image from each iteration, and $300 \operatorname{PSF}^{\prime}\left(\mathrm{X}^{\prime}, \mathrm{Y}^{\prime}\right)$ is PSF in inverse Fourier transform space

301

302 In each iteration of the R-L algorithm (Equation 11), the output image $\left(I_{n+1}\right)$ is estimated 303 restored image array fitting $\mathrm{I}_{n+1}(\mathrm{X}, \mathrm{Y})$ more closely to $\mathrm{I}(\mathrm{X}, \mathrm{Y})$ than the previous $\mathrm{n}_{\text {th }}$ iterations 304 i.e. $I_{n+1}(X, Y) \rightarrow I_{0}(X, Y)$. To test which number of iterations (n) is best for our RD-method, 305 we experiment R-L algorithm with known answer image ( $\left.\mathrm{I}_{0}\right)$ and known PSF-model while 306 monitoring the standard deviation (SD) of the resultant image ( $\left.\mathrm{I}_{\mathrm{n}+1}\right)$ to $\mathrm{I}_{0}$ in every iteration (not 307 shown). It was found that $n \geq 40$ is sufficient to stabilize the $I_{n+1}(X, Y) \rightarrow I_{0}(X, Y)$ at $S D$ of $308 \sim 0.01$. Hence, we consistently apply n=50 for R-L deconvolution in our RD-method.

\subsection{Photometric analysis on the Enormous Cloud Cover using RDD images}

311 In orbits 24 and 25, two largest events of the discontinuity features (ECC) up to latitudinal 312 extent of $\sim 30^{\circ}$ North to $\sim 40^{\circ}$ South (Peralta et al., submitted) have been observed by Akatsuki 313 on 2016 Aug-18 and Aug-27 data. The restoration processes have made these observations 314 more evident for accurate photometric analysis of these events initiating from near the terminator (See Figure 5).

318 The transmittance of the ECC can be measured as follow:

$$
\mathrm{TR}=\frac{\mathrm{I}_{\mathrm{ECC}}}{\mathrm{I}_{\mathrm{BC}}}
$$


where $\mathrm{I}_{\mathrm{ECC}}$ is the intensity in the darker 'Enormous Cloud Cover' (ECC), and $\mathrm{I}_{\mathrm{BC}}$ is the brighter 'Background Clouds' (BC).

323 The region selection for photometric measurements is taken at low latitudes $\left(-5^{\circ}\right.$ to $\left.5^{\circ}\right)$. $\mathrm{I}_{\mathrm{ECC}}$

324 and $\mathrm{I}_{\mathrm{BC}}$ are average radiance of $10^{\circ}$ longitude $\times 10^{\circ}$ latitude square 'box' shaped regions 325 (thereinafter denoted as '10x10-region') each positioned in the optically thick ECC and optically thin $\mathrm{BC}$ regions respectively (Figure 6). They were set to be $10^{\circ}$ longitudinal distances apart while tracking along the propagation of the ECC discontinuity front in subsequent 2 hours interval images. The box regions were shifted longitudinally according to the estimated propagation speed of the ECC $\sim 90 \mathrm{~m} / \mathrm{s}$ (Peralta et al., submitted). The measurements for $\mathrm{I}_{\mathrm{ECC}}$ and $\mathrm{I}_{\mathrm{BC}}$ are summarised in Table 5. The averaged radiance measurements obtained from the respective 10x10-regions for $\mathrm{I}_{\mathrm{ECC}}$ and $\mathrm{I}_{\mathrm{BC}}$ show stable emissions of the $\mathrm{ECC}$ emerging from near the terminator traveling further into the west. This demonstrates the ability of RD process to revert light spreading contamination from the dayside at regions near the terminator. Then, the measurements for all observations as time evolution are plotted in Figure 7 and Figure 8 for Aug-18 and Aug-27 respectively where upper limits of Fogging (Fogging value from Table 4 multiplied by 10) are also being illustrated. To avoid uncertainties in regions too close to the terminator or limb, we select observations of the ECC feature within the region of incident angle $>130^{\circ}$ and emission angle $<75^{\circ}$ for photometric analyses. The discussion on uncertainties associated to the region selection will be further discussed in Section 5.1. 
4.3. Interpretations on aerosol properties of the Enormous Clouds (ECC)

347 The radiative transfer computation as explained in Section 3.2. calculates the wavelength348 dependent $(1.735 \mu \mathrm{m}$ and $2.26 \mu \mathrm{m})$ theoretical transmittance (attenuation) by an additional layer 349 of single-mode aerosols (mode 1, mode 2, mode 2', and mode 3) between 54 to $56 \mathrm{~km}$ altitudes.

350 Extinction cross sections $\left(\mathrm{k}_{\mathrm{ext}}\right)$ incorporated are obtained from the computation for Mie 351 scattering (summarised in Table $3 b$ ). For $2.26 \mu \mathrm{m}$, the relationship between the additional 352 optical thickness $(\tau)$ by different aerosol modes and observed transmittance (TR) can be 353 expressed as the following:

$$
\begin{gathered}
\tau=-\mathrm{A} 3\left(\log \left(\mathrm{TR}_{2.26 \mu \mathrm{m}}^{\mathrm{obs}}\right)\right)^{3}-\mathrm{A} 2 \times\left(\log \left(\mathrm{TR}_{2.26 \mu \mathrm{m}}^{\mathrm{obs}}\right)\right)^{2} \\
-\mathrm{A} 1 \times \log \left(\mathrm{TR}_{2.26 \mu \mathrm{m}}^{\mathrm{obs}}\right)-\mathrm{A} 0
\end{gathered}
$$

354 where $\tau$ is optical depth and $\mathrm{TR}_{2.26 \mu \mathrm{m}}^{\mathrm{obs}}$ is observed transmittance in $2.26 \mu \mathrm{m}$, and the constants (A0, A1, A2, and A3) for different particle size modes are summarised in Table 6 whereas the optical thickness $\tau$ for $1.735 \mu \mathrm{m}$ measurements can then be computed by multiplying $\tau(2.26 \mu \mathrm{m})$ by the ratio of extinction cross sections in Table $3 \mathrm{~b}$.

[Table 6]

The relationships of the theoretical transmissions to the optical depths for different particle modes (mode 1, mode 2, mode 2', and mode 3) are portrayed in Figure 9. Using Equation 13, the optical thicknesses calculated from the measurements $\left(\mathrm{TR}_{2.26 \mu \mathrm{m}}^{\mathrm{obs}}\right)$ obtained from respective 10x10-regions (Table 5) for each observation are summarised in Table 7. 
369 The optical thickness $(\tau)$ obtained together with the extinction cross sections (Table $3 b)$ as

370 calculated from Mie computations for both filters can be used to calculate the column density

371 of particles $(\mathrm{N})$ (summarised in Table 8a). The total column mass of the $\mathrm{H}_{2} \mathrm{SO}_{4}$ droplets can then be calculated (summarised in Table $8 \mathrm{~b}$ ) from $\mathrm{M}=\mathrm{N} \times \mathrm{m}$ ( $\mathrm{M}$ is total column mass) where $\mathrm{m}=\rho \mathrm{V}$ where $\rho$ is $1.834 \mathrm{~g} \mathrm{~cm}-3$ and $\mathrm{V}$ is spherical volume of sulphuric acid droplets where particle size mode's $\overline{\mathrm{r}}$ can be referred to Table $3 \mathrm{a}$.

Figure 10 portrays transmissions measured in the observations $(08-18 / 15: 33$ to $21: 33$; 0827/20:03 to 22:03) over the theoretical transmission curves (mode 1, mode 2, mode2', and mode 3). The observational points for averaged transmissions include error bars that account for spatial variabilities within the measured 10x10-region. Clearly the range of measurements shows that mode 1 cannot be accounted for possible aerosol mode candidate of the ECC. Hence, consideration for mode 1 particles can be exempted from here. This leaves us the possibilities for mode 2, mode 2', and mode 3 to be accounted for the composition of the ECC.

If considering attenuation by additional layer of mode 2 , the overall optical thicknesses of all observations (08-18/15:33 to $21: 33 ; 08-27 / 20: 03$ to $22: 03)$ are in the range of $\tau_{2.26 \mu \mathrm{m}} \sim 11.4-$ 26.7 in $2.26 \mu \mathrm{m}$ (where $\tau_{1.735 \mu \mathrm{m}}$ is 1.385 times larger by referring to wavelength dependent extinction cross section in Table $3 \mathrm{~b}$ ). This means column densities of $\sim 1.3-3.0$ (x108 particles per $\left.\mathrm{cm}_{2}\right)$ amounting to column masses of $\sim 1.0-2.3\left(\mathrm{mg}\right.$ per $\left.\mathrm{cm}_{2}\right)$ are required to suffice the observed additional opacity. 
393 Then, consideration of mode 2' gives optical thicknesses range of $\tau_{2.26 \mu \mathrm{m}} \sim 12.3-28.8$ in

$3942.26 \mu \mathrm{m}$ (where $\tau_{1.735 \mu \mathrm{m}}$ is 1.08 times larger). This results to column densities of $\sim 5.5-12.7$

395 (x107 particles per $\mathrm{cm} 2$ ) amounting to column masses of 1.2 2.7 (mg per $\mathrm{cm}_{2}$ ) are required to 396 suffice the additional optical depths.

397

398 Finally, consideration of mode 3 gives optical thicknesses range of $\tau_{2.26 \mu \mathrm{m}} \sim 5.3-11.3$ in $3992.26 \mu \mathrm{m}$ (where $\tau_{1.735 \mu \mathrm{m}}$ is 1.023 times larger). This leads to column densities of $\sim 4.8-10.3$

400 (x106 particles per $\mathrm{cm} 2$ ) amounting to column masses of 1.8 3.9 (mg per $\mathrm{cm} 2)$ are required to 401 suffice the additional optical depths. Be reminded that these value ranges estimated here are 402 taken from the extreme measurements (lower and upper limits) where the mode value is 403 somewhere within the range for each observation.

404

405 Regardless of particles size, interesting point to note here is that the required amount of column 406 masses to cause the observed optical thicknesses in particles modes (mode 2, mode 2' and 407 mode 3) are not so different. The same amount of sulfuric vapor can condense into particles 408 mode 2, mode 2' and mode 3 to reproduce the observed TR of the ECC. Therefore, all three 409 particles modes (mode 2, mode 2', and mode 3) are possible candidates for constituting of the 410 ECC layer at the current analysis stage. Also, we have to acknowledge that the measurements 411 obtained from the corresponding 10x10-regions in each observation averaged out the spatial 412 variations within each choice of area. This inspired us to continue explore at finer region 413 resolution of $4^{\circ}$ longitude $\times 4^{\circ}$ latitude box region (thereinafter denoted as ' $4 \times 4$-region') for 414 spatial variabilities in each measurement. 


\section{5. Discussions}

\section{5.1. Accuracy of RD method}

420 We evaluate the accuracy of our RD-method by comparing the closeness of our results to the

421 L2B data. We perform this by re-convolving our resultant RDD by model-PSF (denoted as 422 'Convolved-RD' = CRD), and to compare its deviation to the original L2B. The deviation is 423 given by the following equation:

$$
\mathrm{SD}=\sqrt{\frac{\sum|\mathrm{CRD}-\mathrm{L} 2 \mathrm{~B}|^{2}}{\mathrm{~N}}}
$$

424 The ' $\mathrm{SD}^{\prime}$ measures the standard deviation for CRD to L2B (in units of $\mathrm{W}$ m-2 sr-1 $\mu \mathrm{m}-1$ ), and

The plot (Figure 11) shows larger SD values near the terminator indicating some level of influence by dayside still remains in both $2.26 \mu \mathrm{m}$ and $1.735 \mu \mathrm{m}$. The trend shows a rapid decrease until it starts to stabilize from about $130^{\circ}$ incident angles and above at SD of $\sim 0.001$ $0.003 \mathrm{Wm}^{-2} \mathrm{sr}^{-1} \mu \mathrm{m}^{-1}$ in $2.26 \mu \mathrm{m}$ and $\sim 0.003-0.0035 \mathrm{Wm}^{-2} \mathrm{sr}^{-1} \mu \mathrm{m}^{-1}$ in $1.735 \mu \mathrm{m}$. Thus, we perform photometric analysis for regions above $130^{\circ}$ incident angles for better photometric accuracies (defined as 'photometrically stable region'). The small SD in photometrically stable region demonstrates the fidelity of RD-process is good to the observed L2B images. To remark, the SD estimation includes uncertainties associated to various properties: PSF model, plane parallel approximation in radiative transfer for calculating dayside reflectance, and fogging estimation. 


\subsection{Identity of the Enormous Cloud Cover (ECC)}

443 According to the interpretation from Figure 10 (Section 4.3), there is no definitive conclusion

444 of exactly which particle modes are composited in the ECC. However, if assuming ECC is of 445 only mode 3 particles, the Aug-18 event would consist of number density of $~ 5.2-9.2$ (x106 446 particles per $\mathrm{cm}_{2}$ ) which is within the range to that of Aug-27 event giving number density of $447 \sim 4.8-10.3$ (x106 particles per $\mathrm{cm} 2$ ). This could imply that both events are akin to each other given the similar physical properties.

We would like to focus on discussing the data point of $08-18 / 15: 33$ that lies almost exactly on the mode 3 theoretical curve. To further the investigation, we use finer $4 x 4$-regions to probe for spatial variabilities within the $10 \mathrm{x} 10$-region. To specify, $1^{\circ}$ longitude unit and $1^{\circ}$ latitude unit on Venus both corresponds to $\sim 106 \mathrm{~km}$ distance. Therefore, 10x10-region and 4x4-region are $1060 \mathrm{~km} \times 1060 \mathrm{~km}$ and $424 \mathrm{~km}$ x $424 \mathrm{~km}$ respectively on Venus geographical coordinates. The original 10x10-region averages the radiance (hence transmission) value within the area selected which may be missing out detailed transmittance level by finer regions. Bear in mind that the measurements for $\mathrm{I}_{\mathrm{ECC}}$ and $\mathrm{I}_{\mathrm{BC}}$ obtained for regions in $\mathrm{ECC}$ and background cloud (BC) are from separated regions $\left(10^{\circ}\right.$ longitudinal distance apart i.e. $1060 \mathrm{~km}$ apart $)$. This means that to account for all possible spatial variabilities, the transmission measurements have to be

460 all possible combinations of $\mathrm{I}_{\mathrm{ECC}}$ and $\mathrm{I}_{\mathrm{BC}}\left(\mathrm{TR}=\mathrm{I}_{\mathrm{ECC}} / \mathrm{I}_{\mathrm{BC}}\right)$. Thus, our $4 \mathrm{x} 4$-regions scanning 461 within the 10x10-region for both $\mathrm{I}_{\mathrm{ECC}}$ and $\mathrm{I}_{\mathrm{BC}}$ also have to be of all combinations. The key 462 point of incorporating such combinations is because the underlying cloud conditions are 463 unknown and may be changing spatially. Therefore, this motivates our study to be extended into a statistical one to explore all possibilities of aerosol distributions in the ECC. 
465 To do so, we start by moving the $4 \times 4$-region across $10 x 10$-region on each side (latitudinally or 466 longitudinally) for which there can be $6^{\circ}$ shifts resulting in 7 different positions. This gives us 467 a total of 49 different positions when scanning thoroughly in each 10x10-region selected for 468 the ECC and $\mathrm{BC}$ respectively. Then, each $\mathrm{I}_{\mathrm{ECC}}$ and $\mathrm{I}_{\mathrm{BC}}$ measured from $4 x 4$-region would have 49 positions each that gives a total of 2401 possible combinations for $\mathrm{TR}=\mathrm{I}_{\mathrm{ECC}} / \mathrm{I}_{\mathrm{BC}}$.

Interestingly, the scatter plots of 'TR ratios by 4x4-region' (thereinafter denoted as 'TR4') indicate near-parallel alignment to the theoretical transmittance curves regardless of the choice of combinations (Figure 12). This implies the robustness of our method to infer particle modes/sizes by comparing observed transmittance to the theoretical curves. TR4 scattering along the theoretical curve can be related to the variability of optical thicknesses where towards upper right end is more optically thin and towards lower left is more optically thick. On the other hand, the deviation from parallel-ness to the mode 3 theoretical curve is affected by the selection of respective $\mathrm{I}_{\mathrm{ECC}}$ and $\mathrm{I}_{\mathrm{BC}}$ measurements from $4 \mathrm{x} 4$-region where the deviation is indicative of non-mode 3 trend of either the ECC or BC. The slight deviation from parallel alignment to the theoretical curves can be interpreted as two possible contributions: 1. Cause of varying transmissions in the ECC (can be interpreted from IECC statistics); 2. Cause of varying opacities in the $\mathrm{BC}$ (can be interpreted from $\mathrm{I}_{\mathrm{BC}}$ statistics).

The scatters are encompassing well along the mode 3 curve and with small fraction of points touching the mode 2' theoretical curve. Thus, this can be interpreted as the ECC is composite of mostly mode 3 where possible fractional mixings of smaller particles may be considered. 


\subsection{Characterizing the background cloud $(\mathrm{BC})$}

491 To characterize the BC, we consider two categories (with two sub-groups) of the statistical

492 scatter plot by 4x4-regions in Figure 12. To lower the TR4 data points 'Case-L' (L means Low 493 TR4): we need either brighter $\mathrm{I}_{\mathrm{BC}}$ than 'normal' radiances 'Case-LBC' (subscript of 'BC' means 494 lower/higher TR4 by manipulating BC); or darker $\mathrm{I}_{\mathrm{ECC}}$ than normal intensity 'Case-LECC'. In contrast, to increase the data points towards higher TR4 'Case-H' (H means High TR4): we need either darker $\mathrm{I}_{\mathrm{BC}}$ than normal radiances 'Case-HBC'; or brighter $\mathrm{I}_{\mathrm{ECC}}$ than normal 'CaseHECC'. 'Normal' value range here is defined as data points within the standard deviations ( $\mathrm{f}=$ 0.8 where half of the data points lies) of the mean value for the $\mathrm{I}_{\mathrm{BC}}$ and $\mathrm{I}_{\mathrm{ECC}}$ statistics. To note that by manipulating $\mathrm{I}_{\mathrm{BC}}$ or $\mathrm{I}_{\mathrm{ECC}}$ statistics, we can relate them to where their transmission distribution lies on the plot for the BC or ECC respectively. These two cases (of two sub-groups respectively) are summarized in Table 9a-b.

We consider extreme cases of $\mathrm{I}_{\mathrm{BC}}$ data points being brighter than 'normal' (Case-LBC), and $\mathrm{I}_{\mathrm{BC}}$

507 is darker than normal (Case-Hвс). Despite the extremity of the selections, both Case-LBC

508 (Figure 13a) and Case-Hвс (Figure 13b) align well along the mode 3 theoretical curve. Possible explanation is that the lower cloud layer is constantly being heated by infrared radiation originating from the hot lower atmosphere and the ground. The radiation is being absorbed by

511 the cloud particles which drives convection here (Imamura et al.2014; Baker et al., 1998). This

512 process activating the growth of particles into larger size occurs throughout the background 513 cloud. Thus, spatial variations in the BC are almost constantly mode 3 throughout the bright and dark radiances which is being suggested by the statistical analysis. 
516 To compare, we now perform the opposite experiment to evaluate the extreme cases of $\mathrm{I}_{\mathrm{ECC}}$

517 data points being darker than 'normal' (Case-LECC), and $\mathrm{I}_{\mathrm{ECC}}$ is brighter than normal (Case-

518 Hecc). Extreme Case-Lecc shows the scatter points (red) grouping slightly underneath the

519 mode 3 curve (Figure 13a). In contrast, the extreme Case-Hecc illustrates scatter points (red)

520 grouping above the mode 3 curve (Figure 13b). While the preceding Case-Lecc can still be

521 related to mode 3 with possible mixing of smaller mode particles, the latter Case-Hecc is

522 beyond the coverage by mode 3 theoretical curve. This opens the discussions to other

523 considerations to be taken into account to relate to those data points over the theoretical curve

524 range (Case-Hecc).

525

526

[Figure 13]

527

528 5.4. Different approaches to explain observed transmissions

529 5.4.1. Approach 1: Alternative mode 3 size distributions (Mode 3')

530 Here, we consider transmittance by single-mode aerosols with mean radius varied from mode

5313 (named mode 3 '): from $2.0 \mu \mathrm{m}$ to $4.50 \mu \mathrm{m}$ in $0.25 \mu \mathrm{m}$ intervals. For clarity reason, we show

532 in Figure 14 only the theoretical curves for intervals of $0.5 \mu \mathrm{m}$ to avoid crowding the plots in

533 minimal range. The transmittance scatter plots of $08-18 / 15: 33$ are in two groups on the

534 transmission correlation plot. The LECC are grouped on the mode 3' ( $\overline{\mathrm{r}}=2.00 \mu \mathrm{m})$ curve whereas

535 the HECC are still above all mode 3' curves considered.

536

537 Even by modifying the width of size distribution of standard mode 3 particles to be one fifth

538 of the original (uppermost curve) cannot explain the HECC data points. Thus, although some of 
the transmittance scatter points coincide with a part of mode 3' curves, this approach still cannot explain those beyond the mode 3 alternatives' theoretical curves.

\subsubsection{Approach 2: Consideration for different altitude levels of the ECC's aerosols}

545 Then, we consider for different altitude elevations for transmissions by single-mode aerosols

546 of mode 3 in the ECC. The original radiative transfer computation assumed an additional layer

547 of single-mode aerosols at the altitude range $\mathrm{z}=54-56 \mathrm{~km}$ (Figure 10). We vary the radiative

548 transfer calculations for emissions by single-mode aerosols (only mode 3 for this study) at

549 different altitudes varying from $48-60 \mathrm{~km}$ (at $2 \mathrm{~km}$ intervals). The theoretical curves for varying 550 altitudes $(\mathrm{z}=48-60 \mathrm{~km})$ are being expressed in Figure 15 with scatter plot (for all TR4 551 combinations) overlying the curves. Fascinatingly, the scatter plot fits well within the 552 theoretical curves between altitudes at $\mathrm{z}=50 \mathrm{~km}$ (bottom line) and $\mathrm{z}=60 \mathrm{~km}$ (upper line). This 553 suggests consideration for varying altitudes is a promising candidate for further interpretations 554 of the data.

The other interesting thing we can benefit from our statistical analysis is to interpret the

559 distribution probabilities of particles at different altitudes across any region of selection within

560 the ECC. In Figure 16 a-c, we show statistical distribution of $\mathrm{I}_{\mathrm{ECC}}$ starting from constant

561 longitudinal region closest to the discontinuity front shifting across $1^{\circ}$ longitudinal distance 562 away from the front every measurement. These groups of measurements are denoted as ' $\mathrm{LO} \_\mathrm{n}$ ' 563 where 'LO' comes from 'Longitude' and ' $n$ ' is number of constant longitudes ${ }^{\circ}$ away from the 
564 first one 'LO_0'. LO_0 is the closest distance ( 424 km from the front) to the discontinuity

565 front for $\mathrm{I}_{\mathrm{ECC}}$ and furthest for $\mathrm{I}_{\mathrm{BC}}$. To note, $\mathrm{LO}_{-} 0$ is set to be about $4^{\circ}$ longitudinal distance

566 behind the exact front to avoid uncertainties associated to the estimated speed of $90 \mathrm{~m} / \mathrm{s}$ while

567 tracking the ECC front feature (Figure18). Then, $+1^{\circ}$ longitude shift (correspond to $\sim 106 \mathrm{~km}$ )

568 would be denoted as $\mathrm{LO}_{-} 1,+2^{\circ}$ longitude shift is LO_2, and so on. For clarity purpose, we 569 only illustrate altitude curves at $\mathrm{z}=50 \mathrm{~km}$ (bottom line), $\mathrm{z}=54 \mathrm{~km}$ (standard), and $\mathrm{z}=60 \mathrm{~km}$ (upper 570 line) for subsequent statistical analysis.

571

572 We focus on the ECC (Figure 16 a: red points) at LO_0. Interestingly, the (red) scatter plot are

573 all positioned on the outermost edge part of the total combination scatter plots (grey). This suggests that the aerosols at LO_0 (i.e. region closest to discontinuity front) are all at the highest elevation amongst the aerosol distribution in the 10x10-region. This can be interpreted as aerosols are being elevated towards higher altitudes by strong upwelling close to the discontinuity front. Peralta et al. (2019) also observed these discontinuity features using IR1 images (sensitive to altitudes $\mathrm{z} \sim 55-60 \mathrm{~km}$ ). This means that these observations show that the observed discontinuity fronts were being elevated to higher altitudes. According to the analysis using different altitude curves, the data shows that most of the TR4 at higher transmissions align on the $\mathrm{z}=60 \mathrm{~km}$ theoretical curve. In contrast, TR4 in lower transmissions are

582 progressively situated below the $\mathrm{z}=60 \mathrm{~km}$ curve (about $\mathrm{z}=58 \mathrm{~km}$ to $\mathrm{z}=54 \mathrm{~km}$ ). The interpretations

583 from altitudinal variations may suggest aerosols locating at lower altitudes for those in lower transmissions. However, fractional mixing of smaller particles could also be a possibility. 
588 Now, we will analyze the scatter plot progressing from LO_0 to LO_6 which would deliver a sense of altitude variability across regions away from the discontinuity front. To evaluate the transitions of scatter plot from one altitude curve to another, we incorporate RMSD (root-mean-

591 square deviation) measurement for deviations of the observation points (from LO_0 to LO_6)

592 to the respective theoretical altitude curves at: $\mathrm{z}=50 \mathrm{~km}, 52 \mathrm{~km}, 54 \mathrm{~km}, 58 \mathrm{~km}, 56 \mathrm{~km}$, and $60 \mathrm{~km}$

593 (Table 10 and Figure 17). The RMSD is defined as:

$$
\operatorname{RMSD}(\mathrm{z})=\sqrt{\frac{1}{\mathrm{~N}} \sum_{\mathrm{i}=1}^{\mathrm{N}}\left[\mathrm{TR}(\mathrm{i})_{1.735 \mu \mathrm{m}}^{\mathrm{obs}}-\mathrm{TR}_{1.735 \mu \mathrm{m}}^{\text {model }}\left(\mathrm{z}, \mathrm{TR}(\mathrm{i})_{2.26 \mu \mathrm{m}}^{\mathrm{obs}}\right)\right]^{2}}
$$

594 where $\mathrm{TR}_{1.735 \mu \mathrm{m}}^{\mathrm{obs}}$ is observed $1.735 \mu \mathrm{m}$ transmittance, $\mathrm{TR}_{1.735 \mu \mathrm{m}}^{\mathrm{model}}$ is computed by logarithmic595 quartic polynomial approximation (not shown) for respective theoretical altitude (z) curve and observed $2.26 \mu \mathrm{m}$ transmittance $\left(\mathrm{TR}_{2.26 \mu \mathrm{m}}^{\mathrm{obs}}\right)$ where the difference between the observed and model can be obtained, and ' $\mathrm{i}$ ' is for a pair of $\mathrm{TR}_{2.26 \mu \mathrm{m}}^{\mathrm{obs}}$ and $\mathrm{TR}_{1.735 \mu \mathrm{m}}^{\mathrm{obs}}$ number of order of observed transmittance $\mathrm{TR}_{1.735 \mu \mathrm{m}}^{\mathrm{obs}}$ and $\mathrm{N}$ is the total number of elements of observed transmittance $\mathrm{TR}_{1.735 \mu \mathrm{m}}^{\mathrm{obs}}$.

The distribution illustrates transitions from concentration in higher altitude $\mathrm{z}=58 \mathrm{~km}$ at LO_0 to settling at lower altitude $\mathrm{z}=52 \mathrm{~km}$ at LO_3 (Figure 17). At LO_0, the highest probability of concentration is $\mathrm{z}=58 \mathrm{~km}$ as the minimum of RMSD indicates. Then, more aerosol distribution is encompassing around $\mathrm{z}=52-54 \mathrm{~km}$ from LO_3. Particles initially at $\mathrm{z}=58-60 \mathrm{~km}$ altitudes also 
609 descend into the lower altitudes as depicted where RMSD is highest for $\mathrm{z}=58-60 \mathrm{~km}$ curve at

610 LO_3 (Figure 17).

611

612 Assuming the observation at LO_3 is due to temporal change as the discontinuity front

613 propagated away from this region (where spatial variability across $\sim 300 \mathrm{~km}$ is still 614 considerable), this can also be translated as where the particles have sink by $\sim 5 \mathrm{~km}$ (at LO_3)

615 at about $317 \mathrm{~km}$ distance behind the region closest to the front (LO_0). Strong upwelling at the

616 ECC front (LO_0) ascending the mode 3 aerosol aloft as high up as $\mathrm{z}=58 \mathrm{~km}$ altitudes. The

617 ECC propagates about $\sim 20 \mathrm{~m} / \mathrm{s}$ faster than the background super-rotation speed (Peralta et al.,

618 submitted) leaving the uplifted particles behind to sink. (These interpretations are currently

619 based on assumptions where altitudes were the only factor being accounted for and yet to be 620 established). Estimating the particles to sink at LO_3 $(\sim 317 \mathrm{~km}$ away from LO_0), the particles 621 took only $\sim 0.2$ days to descent down to $\mathrm{z}=54 \mathrm{~km}$ while the discontinuity front have already 622 traveled away by $\sim 317 \mathrm{~km}$. For particles to descend by $5 \mathrm{~km}$ in 0.2 days as being observed, it 623 requires $-0.3 \mathrm{~m} / \mathrm{s}$ sinking velocity.

625 However, particle's sedimentation speed is $\sim-0.3 \mathrm{~m} / \mathrm{s}$ from Stokes velocity of $\omega_{\text {sed }}=-\frac{2 \mathrm{ggr}^{2}}{9 \eta} \sim$ $626-0.003 \mathrm{~m} / \mathrm{s}$ where $\mathrm{g} \sim 8.7 \mathrm{~ms}-2$ is gravity acceleration, $\rho \sim 1.8 \times 103 \mathrm{~kg} \mathrm{~m}-3, \mathrm{r}=3.63 \mu \mathrm{m}$ for radius of 627 mode 3 particles, and $\eta \sim 1.5 \times 10-5 \mathrm{~kg} \mathrm{~m}-1 \mathrm{~s}-1$ is the viscosity of $\mathrm{CO}_{2}$ atmosphere (Imamura and 628 Hashimoto., 1998). Sedimentation at this speed would require $\sim 19$ days to descend by $5 \mathrm{~km}$ as 629 being observed. Thus, this could suggest that there could be an additional downwelling motion 630 that accelerates the sinking of the particles to be $\sim 100$ times faster than the Stokes velocity. 631 Look carefully at the boundary of the discontinuity, notice that there is a slight strip of bright 632 radiances (Figure 18). This may hint on strong downwelling force at regions around the 633 discontinuity front. This strong downwelling can propel the aerosols to sink at higher velocity 
634 into the hot lower atmosphere where being evaporated causing higher number density by 635 vapors as illustrated in Figure 19. In subsequent observations however, the bright strip is no

636 longer very visible. This may be due to the increasing convection further in the nightside where 637 downwelling is weakened.

638

639

[Figure 18]

640

641 From LO_3 to LO_6, the transmissions progressively getting lower to below $\sim 0.25$ in $642 \mathrm{TR}(2.26 \mu \mathrm{m})$ and $\sim 0.45 \mathrm{in} \operatorname{TR}(1.735 \mu \mathrm{m})$. This may indicate where accumulated large particles

643 sinking into denser part of the lower cloud deck while accumulating up to column density of $644 \mathrm{~N} \sim(7$ to 8$) \times 10^{6}$ particles $\mathrm{cm}-2$ corresponding to optical thicknesses of $\tau \sim 8$ (i.e. $\left.\mathrm{TR} \sim 0.22\right)$

645 (Figure 17). This would require $\sim 6.90 \mathrm{mg} \mathrm{cm}-2$ amount of vapor to condense into observed 646 optical thickness. While conserving volume, mode 3 conversion to mode 2' particles would 647 increase the optical thickness to $\tau \sim 29.2$. This correspond to lowering the TR down to TR 0.11 648 which is half the transmission level when only mode 3 was considered. Therefore, the 649 consideration for mixing of smaller particles should not be exempted. The RMSD 650 interpretation for proximity to altitudinal variations progressing from LO_0 to LO_6 may 651 suggest that the concentration of particles is located at $\mathrm{z} \sim 52 \mathrm{~km}$ at LO_6 ( $600 \mathrm{~km}$ away from 652 LO_0).

653

654 In overall, the aerosols in high transmissions can only be explained by elevating altitudes. On 655 the other hand, the lower transmissions underlying just below of mode $3(\mathrm{z}=54 \mathrm{~km})$ curve would 656 have two possibilities of considerations: 1. Decreasing altitudes, and/or 2. Mixture of smaller 657 size particles.

658 
660

661

\subsection{Investigation on the spatial variability of the ECC: August-27 data}

662 For August-27 data, we focus on 08-27/20:03 data where its measurement is comparable to 08663 18/15:33. Moving across 20:03/LO_0 to 20:03/LO_6 in Aug-27 (Figure 20a-c), the ECC664 scatter (red) plot shows smooth transition from high altitudes ( $\mathrm{z}=56-58 \mathrm{~km})$ to lower altitudes 665 ( $\mathrm{z}=50 \mathrm{~km})$. The RMSD for LO_0 to LO_6 also explicitly shows transition from high $\mathrm{z}=54$ $66660 \mathrm{~km}$ to low $\mathrm{z}=50 \mathrm{~km}$ (Figure 21). This transitional trend from high altitude curve to low 667 altitude curve can be related to 08-18/15:33 in Section 5.4.2. 20:03/LO_0 to 20:03/LO_6 668 corresponding to $\sim 633.6 \mathrm{~km}$ in distance apart could be displaying two possible interpretations: 669 1. Spatial variation in LO_6 ( 600km across from LO_0) owes to the BC at lower altitudes 670 whereby the ECC would have already travelled away at higher altitudes; 2 . When interpreted 671 from temporal frame, this may be related to the rapid sinking velocity by strong downwelling. 672 The similarity between $08-27 / 20: 03$ to $08-18 / 15: 33$ longitudinal variation (LO_0 to LO_6) 673 shows that the concentration of aerosols begin from locating at higher altitude curves z 56$67458 \mathrm{~km}$, then transiting to the lower altitude curves at $\mathrm{z}=50 \mathrm{~km}$ and $\mathrm{z}=52 \mathrm{~km}$ for $08-27 / 20: 03$ and 675 08-18/15:33 respectively (according to interpretation RMSD plots in Figure 17 and Figure 21).

676

677

678

679

680

681
[Figure 20]

[Figure 21]

[Table 11a]

[Table 11b]

\section{Conclusion}


682 The 'Restoration by Deconvolution' (RD) method has improved the photometric accuracy by 683 true contrast which has enabled photometric studies on the 'Enormous Cloud Cover' (ECC).

684 The ECC events on Aug-18 and Aug-27 2016 are the largest discontinuity features observed 685 by Akatsuki/IR2 with good spatial and temporal tracking. Thorough statistical photometric 686 analyses in RD processed data have been performed on both the Enormous Cloud Cover (ECC) 687 and Background Clouds (BC) to characterize the spatial variabilities of each cloud layer. 688 Utilizing radiative transfer, the spatial variations observed from the ECC have been examined 689 for the distribution of particle modes (mode 1, mode 2, mode 2', and mode 3) where mode 1 690 have been ruled out from our analysis. Other than considering for distribution of smaller 691 particles, possible altitude variations were considered for the observed ECC's spatial variations.

692 The locations of aerosol distribution in the ECC were found to vary starting from higher 693 altitudes $\mathrm{z}=56-58 \mathrm{~km}$ (at LO_0) down to $\mathrm{z}=50-52 \mathrm{~km}$ (at LO_6 i.e. $~ 600 \mathrm{~km}$ away). Also, it has 694 been found that the ECC's discontinuity front experiences both strong upwelling and 695 downwelling motions that sink the large particles at $\sim-0.3 \mathrm{~m} / \mathrm{s}$ that is faster than the 696 sedimentation rate from Stokes velocity $\sim-0.003 \mathrm{~m} / \mathrm{s}$. Such statistical analysis for altitudinal 697 variations of the ECC has never been explored before and hence this could be an opening to 698 more investigations of the peculiar yet intriguing Enormous Cloud in Venusian atmosphere.

699 The mechanism of production and maintenance of the cloud dynamics such as the ECC that 700 absorbs energy from both above and below of the clouds may possibly be related to the 701 maintenance of the super-rotation induced by thermal tides in the cloud top layers. Last but not 702 least, the availability of our processed data will open new doors to exploring identities of other 703 interesting morphologies in hope to explore the cloud dynamics in the lower clouds that could

704 hint on the mechanisms of the unsolved Venus's super-rotation questions.

705

706 Appendix 


\section{Appendix A. Finding dayside F Factor to calibrate RT-calculated dayside}

708 From the development of RD-method, 'convolved models by PSF (CVM)' have already been

709 prepared to simulate observation expectations at dayside $\mathrm{F}=1.0$. Then, we can calibrate for the

710 actual dayside $\mathrm{F}$ factor by comparing the light spreading in CVM (at $\mathrm{F}=1.0$ ) to L2B

711 observations. The calibration was performed by selecting a 25 -pixels2 size 'box' region at

712 constant pixel-distance away from the Venus's dayside limb for both CVM and L2B images

713 where radiance values in the selected 'box' were averaged for CVM and L2B respectively.

714 The $\mathrm{F}$ factor for $2.26 \mu \mathrm{m}$ is hence:

$$
\mathrm{F}(2.26 \mu \mathrm{m})=\sum \overline{\overline{\mathrm{CVM}}}
$$

716

717 where $\overline{\mathrm{CVM}}$ and $\overline{\mathrm{L} 2 \mathrm{~B}}$ are averaged radiances from the 25-pixels2 box at constant pixel718 distances away from the Venus's dayside limb.

Then, the $\mathrm{F}$ factor for $1.735 \mu \mathrm{m}$ can be computed as:

$$
\mathrm{C}=\sum \frac{\overline{\mathrm{MDD}_{1.735 \mu \mathrm{m}}}}{\overline{\mathrm{MDD}_{2.26 \mu \mathrm{m}}}}
$$

$$
\mathrm{R}=\frac{\mathrm{k}_{2.26 \mu \mathrm{m}}}{\mathrm{C}}
$$

$$
\mathrm{F}(1.735 \mu \mathrm{m})=\mathrm{R} \times \mathrm{F}(2.26 \mu \mathrm{m})
$$

724 where $\overline{\mathrm{MDD}_{2.26 \mu \mathrm{m}}}$ and $\overline{\mathrm{MDD}_{1.735 \mu \mathrm{m}}}$ are mean radiances of total integrated flux from the

725 Model Daysides (MDD) for $2.26 \mu \mathrm{m}$ and $1.735 \mu \mathrm{m}$ filter respectively, and $\mathrm{k}_{2.26 \mu \mathrm{m}}$ is $\mathrm{k}_{2.26 \mu \mathrm{m}}=$ $\left(8.4277 \times 10^{-4}\right) \alpha^{2}-\left(2.2731 \times 10^{-1}\right)+17.979$ from Satoh et al., (submitted) which is a calibration factor to adjust $2.26 \mu \mathrm{m}$ dayside intensities into $1.735 \mu \mathrm{m}$ filter.

729 Finally, the corrected Model Daysides (MDD') can be expressed as the following 


$$
\mathrm{MDD}_{2.26 \mu \mathrm{m}}^{\prime}=\mathrm{MDD}_{2.26 \mu \mathrm{m}} \times \mathrm{F}(2.26 \mu \mathrm{m})
$$

$$
\mathrm{MDD}_{1.735 \mu \mathrm{m}}^{\prime}=\mathrm{MDD}_{1.735 \mu \mathrm{m}} \times \mathrm{F}(1.735 \mu \mathrm{m})
$$

Table A summarizes the Dayside F factors obtained from the above calculations.

\section{Appendix B. Photometric restoration by RD and RS methods}

736 Synthetic models simulating Venus observations under influence by camera's conditions (PSF, dayside contamination, and saturation) are generated to test restorative strengths of the two methods: RD and RS. For this test, we use 2016/08/18 15h RD-restored resultant as nightside answer image (RRD), radiative transfer calculated dayside (RTD), and known model-PSF. Then, we can synthesize the full Venus disk (MDV) being convolved by model-PSF to simulate the typical observation condition by IR2 camera. RD and RS processes are performed on the convolved image where the results can be compared with the known answer image using scatter plots of resultants to answer image (Figure A1).

To clarify here, there are two Restoration-by-Subtraction (RS) methods: Restoration-bySubtraction 'Subtraction-only' (RSS) (Satoh et al., submitted), and Restoration-by-Subtraction 'using PSF' (RSP). Both RSS and RSP incorporates the same concept where both methods minimize contamination by dayside PSF. Thus, RSP performed here intends to simulate RSS in the synthetic case. RSS performs subtraction by differentiating two filters (Section 3.3). On

750 the other hand, RSP simulates the dayside light spread by convolving the radiative transfer calculated dayside ('Convolved Radiative Transfer Dayside’ = 'CRTD') by model-PSF. Then, the image is restored by subtraction (RSP): CLN $=$ L2B - CRTD. 
RSP-answer scatter plot giving $\sim 0.7(<1)$ slope indicates inability of RS method to recover

755

756

757

758

759

760

761

762

763

764

765

766

767

768

769

770

771

772

773

774

775

776

777

778 light scattered away from each pixel due to PSF. For bright pixels, RS method does not recover light spread by PSF into adjacent pixels. In dark pixels, RS method cannot remove remaining radiances of light spread by adjacent brighter pixels after subtraction being ceased when overall minimum contamination is achieved. This results in the y-intercept offset value of $\sim 0.005$ where ideal restoration should bring the y-intercept closer to 0 .

In contrast, RD-answer scatter plot's slope of $0.98(\sim 1)$ shows the working of deconvolution to recover light spread into adjacent pixels back to its original source. Imagine a bright pixel in the nightside, RD works by re-collecting light spread by PSF into adjacent pixels back to its original position. As for a dark pixel in the nightside, the influence of light spread by adjacent brighter pixels can be removed during deconvolution process. This gives the almost $\sim 0 \mathrm{y}-$ intercept in the RD-answer scatter plot.

Figure A2 illustrates the comparison of both RD and RS method. The RD-RS scatter plot illustrates higher slope $\sim 1.3(>1)$ indicating RD has more photometry restored for bright pixels when compared to RSS. The negative y-intercept also shows that darker pixels in nightside are better recovered from influences of adjacent pixels in RD to that of RS.

[Figure A1]

[Figure A2]

\section{Declarations}

\section{Ethics approval and consent to participate}

Not applicable 


\section{Consent for publication}

$780 \quad$ Not applicable

781

782 List of abbreviations

783 PV: Pioneer Venus

784 LCPS: Cloud Particle Size Spectrometer

785 NIMS: Near Infrared Mapping Spectrometer

786 VIRTIS: Visible and Infrared Thermal Imaging Spectrometer

787 VEx: Venus Express

788 PSF: point spread function

789 RMSD: Root-mean-square deviation

790 ROI: Region of Interest

791 RSS: Restoration by Simple Subtraction

792 RD: Restoration by Deconvolution

793 MDD: Model Dayside Disk

794 CLN: Cleaned Night

795 Model Dayside + Nightside: MDN

796 CVM: Convolved Model

797 COM: Combined frame

798 RDD: RD-processed Data

799 ECC: Enormous Cloud Cover

800 BC: Background Cloud

801 SD: Standard Deviation

802 R-L: Richardson-Lucy

803 
804 Availability of data and materials

805 The authors are going to archive and publish the RD processed data to make available for 806 public use.

807

808 Competing Interests

809 None

810

\section{Funding}

812 CWV was supported by the Japanese Government Monbukagakusho (MEXT) scholarship to 813 conduct this study.

814

\section{Authors' contributions}

$816 \mathrm{CWV}$ is responsible for all elements of this manuscript. TS calibrated the temperature

817 dependent behavior of IR2 data and has supervised CWV in conduct of this study within

818 doctoral course. TMS provided radiative transfer calculations for dayside reflectance. TH 819 corrected the image navigation of the spacecraft using limb fitting technique.

820

\section{Acknowledgements}

822 The authors are grateful to all members of Akatsuki (Planet-C) to have accomplished

823 successful observations of Venus. Special thanks to Dr. J. Peralta who has been reliable to 824 discuss about the intriguing findings in this paper. Furthermore, the authors would like to 825 express gratitude to Dr. K. McGouldrick, Dr. E. Young, and Dr. S. Limaye who have been 826 contributive to Akatsuki mission and scientific discussions. Not to mention, Dr. T. Oba has 827 also been supportive in sharing his knowledge on deconvolution technique performed on 828 Hinode observations. Finally, the authors are also indebted to Prof. M. Kimata, Ritsumeikan 
829 University and manufacturers of IR2 (Sumitomo Heavy Industries, Ltd., Mitsubishi Electronic

830 Company MELCO, Nikon, Magoshi, and Fujitoku and Barr Associates) for the development

831 of the Akatsuki/IR2 camera.

832

833 Authors' information

834 TS is the Principle Investigator of Akatsuki/IR2.

835

836 Endnotes

837 None

838

839 References

840 Belton MJS et al (1991) Images from Galileo of the Venus Cloud Deck. Science 253:1531-

841 1536. https://doi.org/10.1126/science.253.5027.1531

842

843 Baker RD, Schubert G, Jones PW (1998) Cloud-level penetrative compressible convection in

844 the Venus atmosphere. Journal of Atmospheric Sciences 55, 3-18.

845

846 Carlson R et al (1991) Galileo infrared imaging spectrometer measurements at Venus. Science $847 \quad 253,1541-1548$.

848

849 Carlson R et al (1993) Variations in Venus cloud particle properties: a new view of Venus's

850 cloud morphology as observed by Galileo near-infrared mapping spectrometer. Planetary and $851 \quad$ Space Science 41: 477-485.

852 
853 Esposito LW, Knollenberg RG, Marov MY, Toon OB, and Turco RP (1983) The clouds and 854 hazes of Venus. In Venus, edited by Hunten, DM et al, The University of Arizona Press, Tucson, 855 pp. $484-564$.

856

857 Grinspoon DH et al (1993) Probing Venus's cloud structure with Galileo NIMS, Planetary and 858 Space Science 41(7), 515-542.

859

860 Haus R, Kappel D, Arnold G (2015) Lower atmosphere minor gas abundances as retrieved

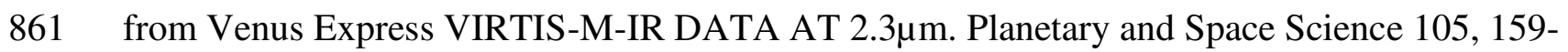
862174.

863

864 Hueso R, Peralta J, Sanchez-Lavega A (2012) Accessing the long-term variability of Venus 865 winds at cloud level from VIRTIS-Venus Express. Icarus 217, 585-598.

866

867 Horinouchi T (2017) Equatorial jet in the lower to middle cloud layer of Venus revealed by 868 Akatsuki. Nature Geoscience. 10, 646-651.

869

870 Imamura T, Hashimoto GL (1998) Venus cloud formation in the meridional circulation.

871 Journal of Geophysical Research 103, 31349-31366.

872

873 Imamura T, Higuchi T, Maejima Y., Takagi M, Sugimoto N, Ikeda K, Ando H (2014) Inverse 874 insolation dependence of Venus's cloud-level convection. Icarus 228, 181-118.

875 
876 Knollenberg RG and Hunten DM (1980) The microphysics of the clouds of Venus: Results of

877 the Pioneer Venus Particle Size Spectrometer experiment. Journal of Geophysical Research., 878 85(A13), 8039-8058.

879

880 McGouldrick K, Momary TW, Baines KH, and Grinspoon DH (2012) Quantification of middle 881 and lower cloud variability and mesoscale dynamics from Venus Express/VIRTIS observations 882 at $1.74 \mu \mathrm{m}$. Icarus $217,615-628$.

883

884 McGouldrick K (2017) Effects of variation in coagulation and photochemistry parameters on 885 the particle size distributions in the Venus clouds. Earth Planets Space 69:161. 886 https://doi.org/10.1186/s40623-017-0744-X

887

888 Murakami S, Satoh T, McGouldrick K, Yamamoto Y, Hashimoto GL (2018) Venus Climate 889 Orbiter Akatsuki IR2 calibrated data v1.0, VCO-V-UVI-3-CDR-V1.0. In: NASA Planetary 890 Data System. https://doi.org/10.17597/ISAS.DARTS/VCO-00003.

891

892 Palmer KF and Williams D (1975) Optical Constants of Sulfuric Acid; Applications to the 893 Clouds of Venus?. Applied Optics Vol. 14, No.1.

894

895 Peralta J et al (2018) Nightside Winds at the Lower Clouds of Venus with Akatsuki/IR2: 896 Longitudinal, Local Time, and Decadal Variations from Comparison with Previous 897 Measurements. Astrophysical Journal Supplement Series. 239:29 (17pp). 898 https://doi.org/10.3847/1538-4365/aae844 899 
900 Peralta J et al (2019a) Morphologies and Dynamics of Venus's Middle Clouds with

901 Akatsuki/IR1. Geophysical Research Letters. 46(5), 2399-2407.

902

903 Peralta $\mathbf{J}$ et al (submitted) A long-lived disruption on the lower clouds of Venus. Submitted to 904 Geophysical Research Letters.

905

906 Pollack JB et al (1979) Nature of the ultraviolet absorber in the Venus clouds - Inferences

907 based on Pioneer Venus data $205(4401) \cdot 76-79$.

908 https://doi.org/10.1126/science.205.4401.76

909

910 Ragent B et al (1985) Particulate matter in the Venus atmosphere. Adv. Space Res. Vol 5, 911 No.11, p85-115.

912

913 Satoh T et al (2016) Development and in-flight calibration of IR2: 2- $\mu \mathrm{m}$ camera onboard

914 Japan's Venus orbiter, Akatsuki. Earth Planets Space 68:74. https://doi.org/10.1186/s40623$915 \quad 016-0451-z$

916

917 Satoh T et al (2017) Performance of Akatsuki/IR2 in Venus orbit: the first year. Earth Planets

918 Space 69:154. https://doi.org/10.1186/s40623-017-0736-x

919

920 Satoh $\mathrm{T}$ et al (submitted). Venus nightside photometry with "cleaned" Akatsuki/IR2 data: 921 Aerosol properties and variations of carbon monoxide. Submitted to Icarus.

922

923 Sato TM et al (2020) Dayside cloud top structure of Venus retrieved from Akatsuki IR2 924 observations. Icarus 345, 113-682. 
926 Seiff, A (1982) Dynamical implocations of the observed thermal contrast in Venus's upper 927 atmosphere. Icarus 51, 574-592.

928

929 Stamnes K, Tsay SC, Wiscombe WJ, Jayaweera K (1988) Numerically stable algorithm for 930 discrete-ordinate-method radiative transfer in multiple scattering and emitting layered media. 931 Applied Optics 27, 2502-2509.

932

933 Titov DV, Ignatiev NI, McGouldrick K, Wilquet V, Wilson CF (2018) Clouds and Hazes of 934 Venus. Space Science Reviews, 214:126

935 
936

937

938

939

940

941

942

943

944

945

946

947

948

949

950

951

952

953

954

955

956

957

958

959

960

\section{Figure Legends}

Figure 1 -Level 2b (L2B) data example showing influence of dayside light spread due to IR2 camera's point spread function (PSF) and saturation. Saturated pixels were given $-100 \mathrm{~W}$ m-2

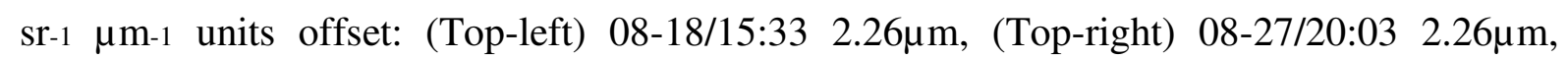

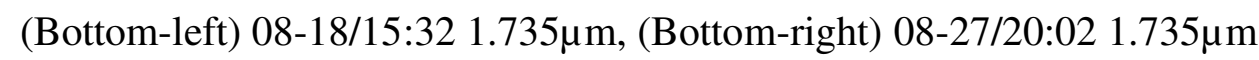

Figure 2 - (a) Image resultants from Step 2 to Step 5 of RD method i.e. (Step 2) MDD \& CLN, (Step 3) CVM, (Step 4) COM, and (Step 5) RDD; (b) Image preparations from Step 2 to Step 5 of RD method i.e. (Step 2) MDD (x 0.01 to scale to other images) \& CLN, (Step 3) CVM, (Step 4) COM, and (Step 5) RDD.

Figure 3 - Loss to Off-Disk/Total Flux plot against spacecraft distance from Venus in Logarithmic scale for $2.02 \mu \mathrm{m}$ dayside observation data (L2B) below $45^{\circ}$ phase angle (orbit 14,15, and 16), and synthetic dayside convolved by model-PSF: Red (orbit 14), Yellow (orbit 15), Orange (orbit 16).

Figure 4 - (Left) Example L2B data (2016-08-18 21:33) in 2.26 $\mu \mathrm{m}$ showing image was trimmed by [129:896, 385:1024] 'Region of Interest' (ROI). The image is in the original orientation of the downlink data where North is downwards. (Right) Preparation of $2048 \mathrm{x}$ 2048 pixels2 size frame to accommodate simulation of light spread by PSF outside ROI frame and saturated pixels being replaced. In this example, the larger canvas outside ROI frame enables loss photometry up to $\sim 14 \%$ to be recovered where light spreading can be pulled back by deconvolution. The yellow arrows point on the PSF tail extension spreading light outside the ROI frame. 
961 Figure 5 - RDD image example in $2.26 \mu \mathrm{m}$ where the measurements for radiance contrast will

962 be illustrated in Figure 8: (a) $2.26 \mu \mathrm{m}$; (b) $1.735 \mu \mathrm{m}$

963

964 Figure $6-10^{\circ}$ longitude $\times 10^{\circ}$ latitude pixel 2 size 'box' to obtain average radiance within the 965 selected region both in the optically thick enormous cloud and bright background $\left(10^{\circ}\right.$ 966 longitude apart) to measure the averaged transmittance of radiation through the enormous cloud 967 feature. The boxes are shifted longitudinally by estimated speed in between the 2-hr 968 observations. Image examples shown here are 2-hr intervals of limb darkening corrected RD969 restored data where the wind speed of the enormous cloud is estimated at $90 \mathrm{~m} / \mathrm{s}$. (a) 08 $970 \quad 18 / 15: 33$ to $21: 33$; (b) $08-27 / 20: 03$ to $22: 03$

971

972 Figure 7 - Average radiances of 'box region' in ECC and BC as selected in Figure 6a obtained 973 for RDD images. Fogging factor x 10 range is included: (a) Aug-18 in 2.26 $\mu$ m; (b) Aug-18 in $974 \quad 1.735 \mu \mathrm{m}$

975

976 Figure 8 - Average radiances of 'box region' in ECC and BC as selected in Figure 6b obtained 977 for RDD images. Fogging factor x 10 range is included: (a) Aug-27 in 2.26 $\mu \mathrm{m}$; (b) Aug-27 in $978 \quad 1.735 \mu \mathrm{m}$

979

980 Figure 9 - Radiative transfer calculation for transmittance by additional optical thickness of 981 single-mode aerosols (mode 1, mode 2, mode 2', and mode 3) between 54 to $56 \mathrm{~km}$ altitude in: 982 (a) $2.26 \mu \mathrm{m}$; (b) $1.735 \mu \mathrm{m}$.

983 
984 Figure 10 - Observed Transmittance (TR) with associated uncertainty range plotting over theoretical Transmittance (TR) computed by radiative transfer for different particle size modes

986 (mode 1, mode 2, mode 2', mode 3).

987

988 Figure 11 - Standard deviation of CRD to L2B images in bins of $10^{\circ}$ incident angles for: (a)

$2.26 \mu \mathrm{m}$; (b) $1.735 \mu \mathrm{m}$. There are no data for incident angles before $120^{\circ}$ due to saturation.

990

991 Figure 12- Total combinations of 4x4-regions' transmission ratios plotted over particle mode

992 theoretical curves (mode 1, mode 2, mode 2' mode 3 ) showing alignment around mode 3 and 993 slightly reaching mode 2' theoretical curves.

994

995 Figure 13 - (a) Case-L in which to lower the transmissions. This is divided into two sub-groups. 996 'Case- $\mathrm{L}(\mathrm{BC})$ ' where $\mathrm{I}_{\mathrm{BC}}$ is brighter than 'normal' intensity and 'Case $\mathrm{L}(\mathrm{ECC})$ ' where $\mathrm{I}_{\mathrm{ECC}}$ is 997 darker than normal intensity; (b) Case-H in which to increase to higher transmissions. This is 998 divided into two sub-groups. 'Case- $\mathrm{H}(\mathrm{BC})$ ' where $\mathrm{I}_{\mathrm{BC}}$ is darker than 'normal' intensity and 999 'Case L(ECC)' where $\mathrm{I}_{\mathrm{ECC}}$ is brighter than normal intensity

1000

1001 Figure 14 - Theoretical transmittance calculated for mode 3' ranging from $\bar{r}=2.0 \mu \mathrm{m}$ to $4.5 \mu \mathrm{m}$ 1002 in $0.25 \mu \mathrm{m}$ intervals, and one fifth width of size distribution of mode 3 to the original denoted 1003 as ' $3.65 \mu \mathrm{m} \_$sig', and mode 3 is $3.65 \mu \mathrm{m}$ mean radius. (Red Dots) are from previous Figure $13 \mathrm{a}$ 1004 and $13 \mathrm{~b}$ representing $\mathrm{I}_{\mathrm{ECC}}$ points.

1005

1006 Figure 15 - Radiative transfer calculations for emissions by single-mode aerosols (only mode 10073 for this study) at different altitudes varying from $48-60 \mathrm{~km}$ (at $2 \mathrm{~km}$ intervals). 
1009 Figure 16 - Combination scatter plots within 10x10region with resolution of 4x4-region 1010 statistical analysis. (Red) Transmission distribution of the Enormous Cloud Cover 'ECC'.

1011 (Blue) Transmission distribution of the Background Cloud 'BC' in 08-18/15:33: (a) LO_0,

1012 (b) LO_3, (c) LO_6

1013

1014 Figure 17 - RMSD for longitudinal distance away from discontinuity front (spatial variation):

1015 08-18/15:33. Left y-axis is RMSD and Right y-axis optical depths.

1016

1017 Figure 18-Close up image of RD-data (blue curve line) discontinuity front, (yellow arrow) 1018 bright downwelling features. (a) for 08-18/15:33, 17:33, 19:33, and 21:33. Note on the bright 1019 strip just after the front is most prominent at 15:33, then decreasing gradually in radiance to 1020 21:33; (b) 08-27/20:03 and 22:03.

1021

1022 Figure 19 - Schematics of ECC

1023

1024 Figure 20 - Combination scatter plots within 10x10region with resolution of 4x4-region 1025 statistical analysis. (Red) TR4 of the Enormous Cloud Cover 'ECC'. (Blue) Transmission distribution of the Background Cloud 'BC' in 08-27/20:03: (a) LO_0, (b) LO_3, (c) LO_6

1028 Figure 21 - RMSD for longitudinal distance away from discontinuity front (spatial 1029 variation): 08-27/20:03. Left y-axis is RMSD and Right y-axis optical depths. 1030

1031 Figure A1- Comparison of restorative strengths in RD and RSS method. (Green) RD-answer 1032 scatter plot and (Red) RSS-answer scatter plot. 
1033 Figure A2 - RD-RSS scatter plot showing that RD restoration could restore more loss

1034 photometry than RSS method

1035

1036 Tables

1037 Table 1 - Summary of Venus observations in $2.26 \mu \mathrm{m}$ and $1.735 \mu \mathrm{m}$ filters used in this study.

1038

a. $2.26 \mu \mathrm{m} \mathrm{L} 2 \mathrm{~B}$ data

1039

b. $1.735 \mu \mathrm{m}$ L2B data

1040

1041 Table 2 - Summary of denotations used for RD-method in this paper

1042

1043 Table 3 - Aerosol properties set in radiative transfer computation

1044 b. Wavelength dependent cross section extinction cross sections $\left(\mathrm{k}_{\text {ext }}\right)$ and single scattering 1045 albedos $(\omega)$ for particles in $2.26 \mu \mathrm{m}$ and $1.735 \mu \mathrm{m}$

1046

1047 Table 4 - Calibration for Fogging values

1048 a. $2.26 \mu \mathrm{m}$ filter

1049 b. $1.735 \mu \mathrm{m}$ filter

1050

1051 Table 5 - Transmittance of the Enormous cloud measured within 'photometrically-stable

1052 region (incident angle $>130^{\circ}$ )' for Aug-18 IECC and IBC are averaged intensity of the box

1053 region in $\mathrm{Wm}^{-2} \mathrm{sr}^{-1} \mu \mathrm{m}^{-1}$ unit, whilst TR is transmittance.

1054

1055 Table 6 - Coefficients for computing $\tau$ from Transmittance

1056 
Table 7 - Optical thickness of the Enormous cloud measured within 'photometrically-stable region (incident angle $>130^{\circ}$ )

1059

1060 Table 8 - Calculations for $\mathrm{H}_{2} \mathrm{SO}_{4}$ particles' properties observed in the ECC measured within

1061 'photometrically-stable region (incident angle $>130^{\circ}$ )' for 10x10-region.

1062

1063

a. Column Density

b. Column Mass

1064

1065 Table 9 - Statistical analysis for IECC and IBC combinations in 4x4-regions

1066 a. Tabulation of all possible combinations of the $\left(\mathrm{TR}=\mathrm{I}_{\mathrm{ECC}} / \mathrm{I}_{\mathrm{BC}}\right)$ measurements for Case $\mathrm{L}$ and Case H. $\mathrm{f}=0.8$ is chosen to include about half the data points and sigma is the standard 1068 deviation of TR4.

1069 b. Extreme cases of I_BC and I_ECC to lower TR4 (Case-L) or to increase transmissions to 1070 higher TR4 (Case-H)

1071

1072 Table 10 - RMSD from altitude curves and optical thickness range for longitudinal variation 1073 from LO_0 to LO_6 measurements for 08-18/33

1074 a. RMSD of $\mathrm{TR}_{1.735 \mu \mathrm{m}}^{\mathrm{obs}}$ to $\mathrm{TR}_{1.735 \mu \mathrm{m}}^{\text {model }}$ from different altitude curves from LO_0 to LO_6

1075 b. Optical Depths range where 'Min' is minimum, 'Max' is maximum values of $\tau$

1077 Table 11 - RMSD from altitude curves and optical thickness range for longitudinal variation 1078 from LO_0 to LO_6 measurements for 08-27/20:02

1079 a. RMSD of $\mathrm{TR}_{1.735 \mu \mathrm{m}}^{\mathrm{obs}}$ to $\mathrm{TR}_{1.735 \mu \mathrm{m}}^{\text {model }}$ from different altitude curves from LO_0 to LO_6 
1082 Table A - Dayside Calibration Factors

1083 a. $2.26 \mu \mathrm{m}$

1084 b. $1.735 \mu \mathrm{m}$ 


\section{Figures}

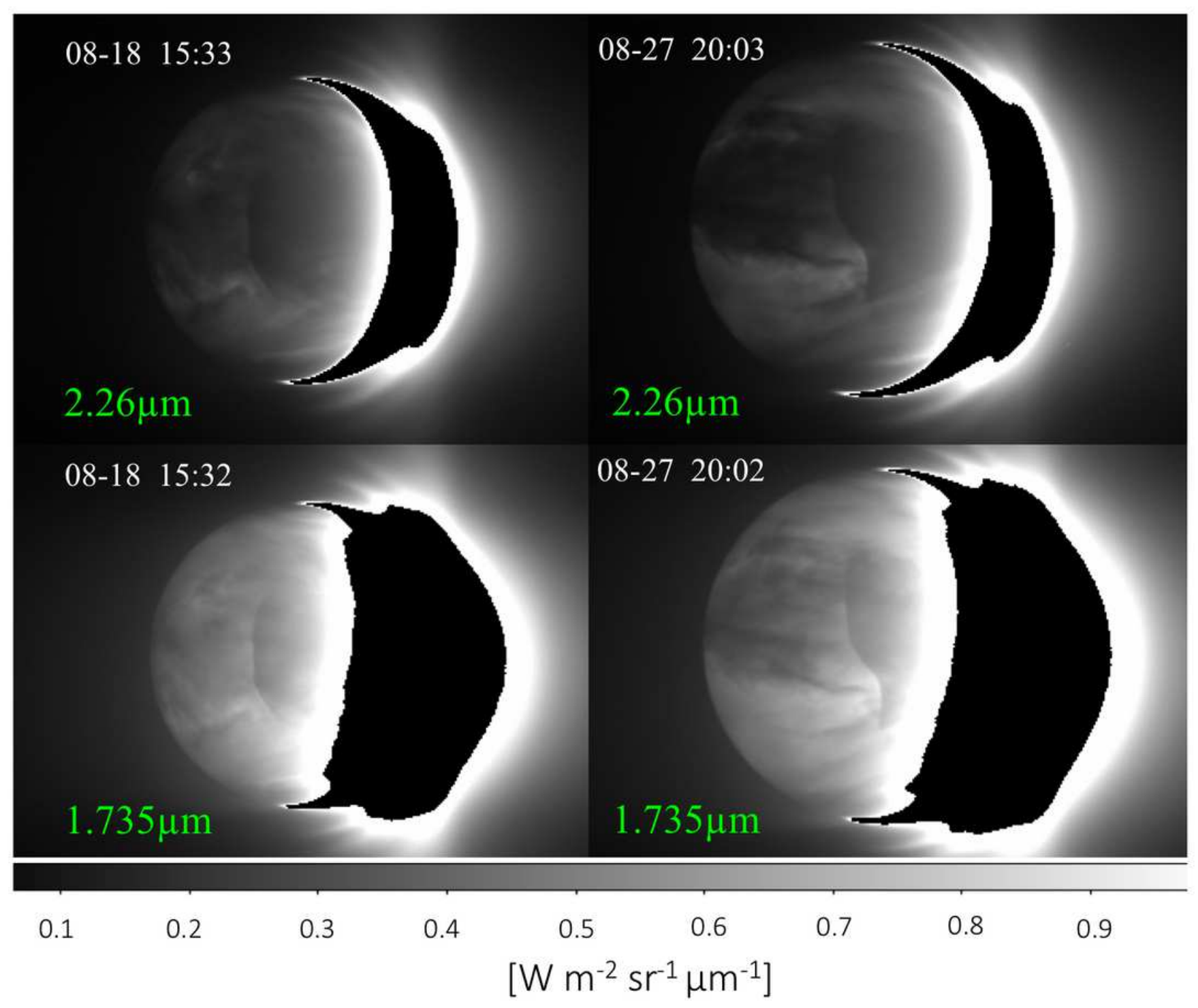

Figure 1

Level 2b (L2B) data example showing influence of dayside light spread due to IR2 camera's point spread function (PSF) and saturation. Saturated pixels were given -100 W m-2 sr-1 $\mu \mathrm{m}-1$ units offset: (Top-left)

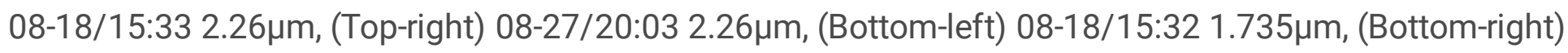

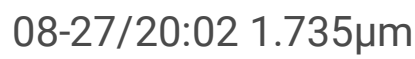


(a) Flowchart of RD-method from Step 1 to Step 5

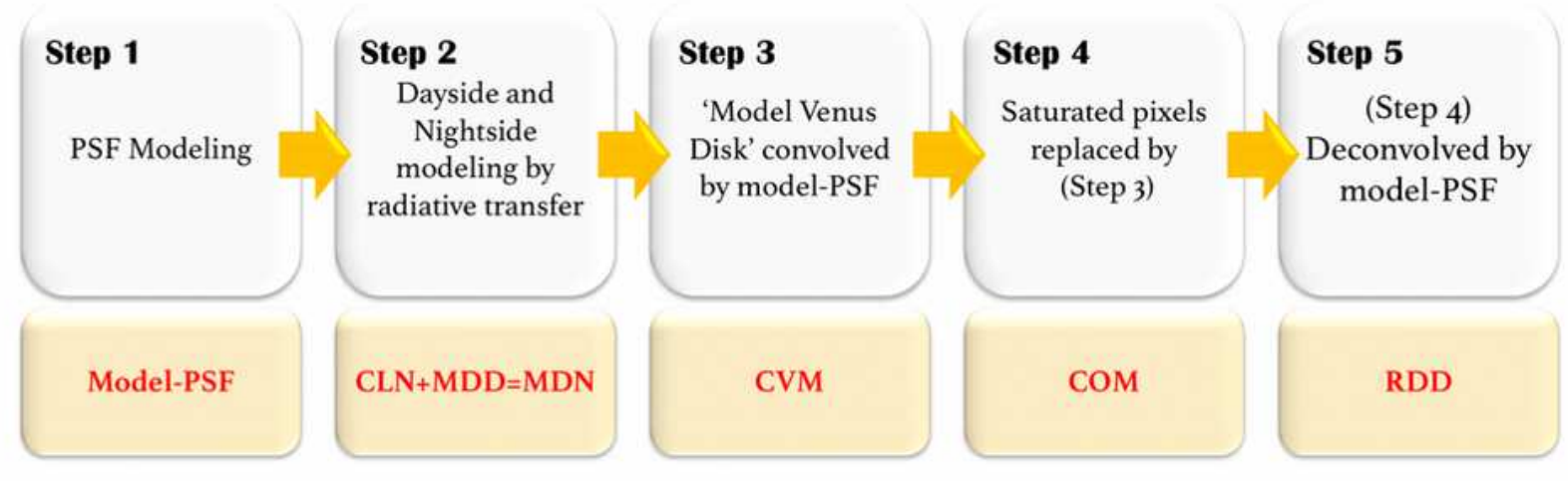

(b) Sequential Image Preparations from Step 1 to Step 5

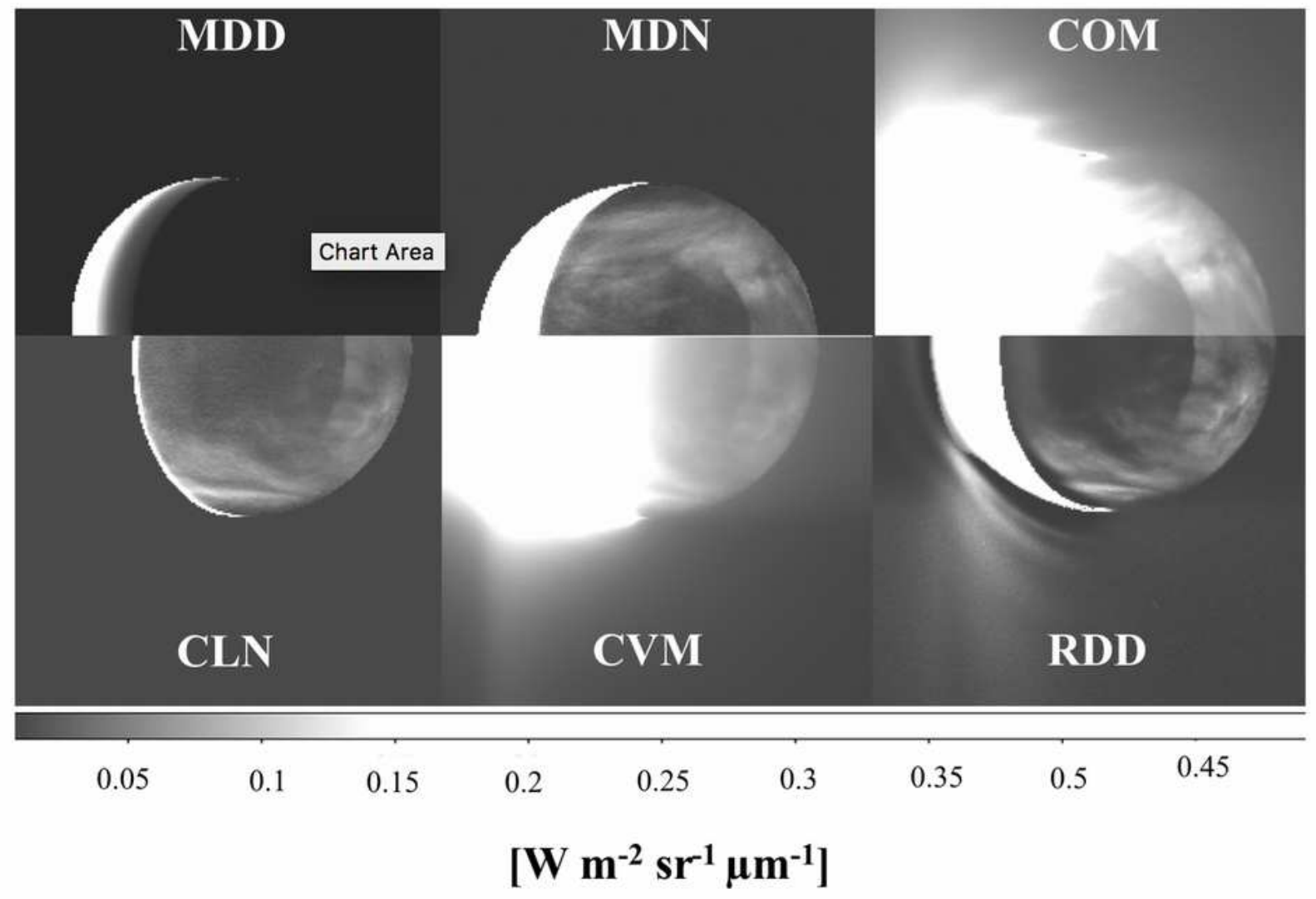

Figure 2

(a) Image resultants from Step 2 to Step 5 of RD method i.e. (Step 2) MDD \& CLN, (Step 3) CVM, (Step 4) COM, and (Step 5) RDD; (b) Image preparations from Step 2 to Step 5 of RD method i.e. (Step 2) MDD (x 0.01 to scale to other images) \& CLN, (Step 3) CVM, (Step 4) COM, and (Step 5) RDD. 


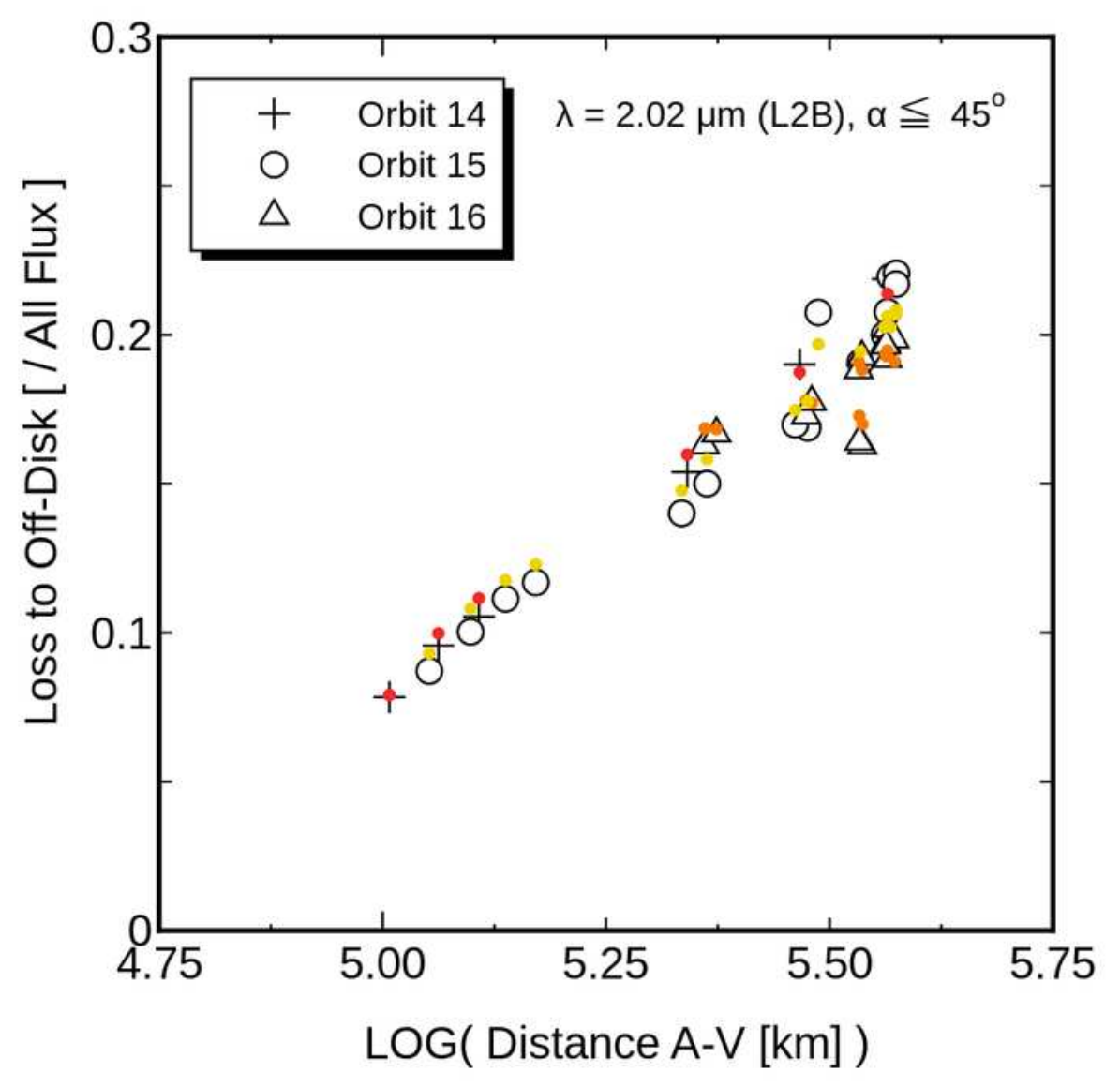

Figure 3

Loss to Off-Disk/Total Flux plot against spacecraft distance from Venus in Logarithmic scale for $2.02 \mu \mathrm{m}$ dayside observation data (L2B) below $45^{\circ}$ phase angle (orbit 14,15, and 16), and synthetic dayside convolved by model-PSF: Red (orbit 14), Yellow (orbit 15), Orange (orbit 16). 


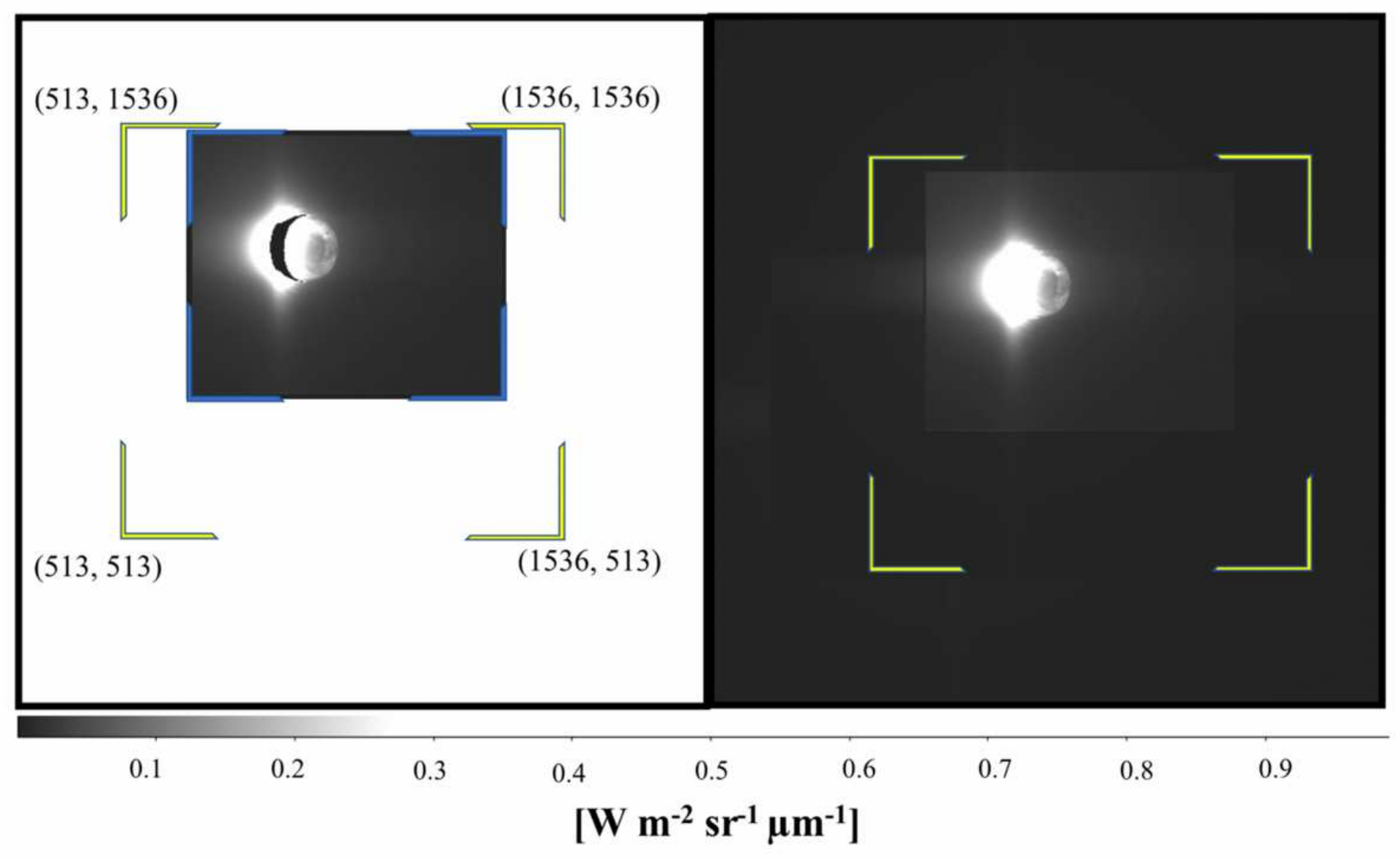

Figure 4

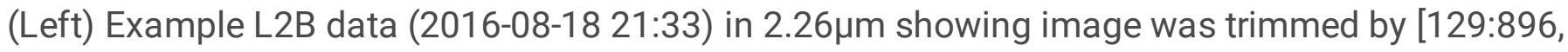
$385: 1024]$ 'Region of Interest' (ROI). The image is in the original orientation of the downlink data where North is downwards. (Right) Preparation of 2048 x 2048 pixels2 size frame to accommodate simulation of light spread by PSF outside ROI frame and saturated pixels being replaced. In this example, the larger canvas outside ROI frame enables loss photometry up to $\sim 14 \%$ to be recovered where light spreading can be pulled back by deconvolution. The yellow arrows point on the PSF tail extension spreading light outside the ROI frame. 
(a) RDD: $2.26 \mu \mathrm{m}$

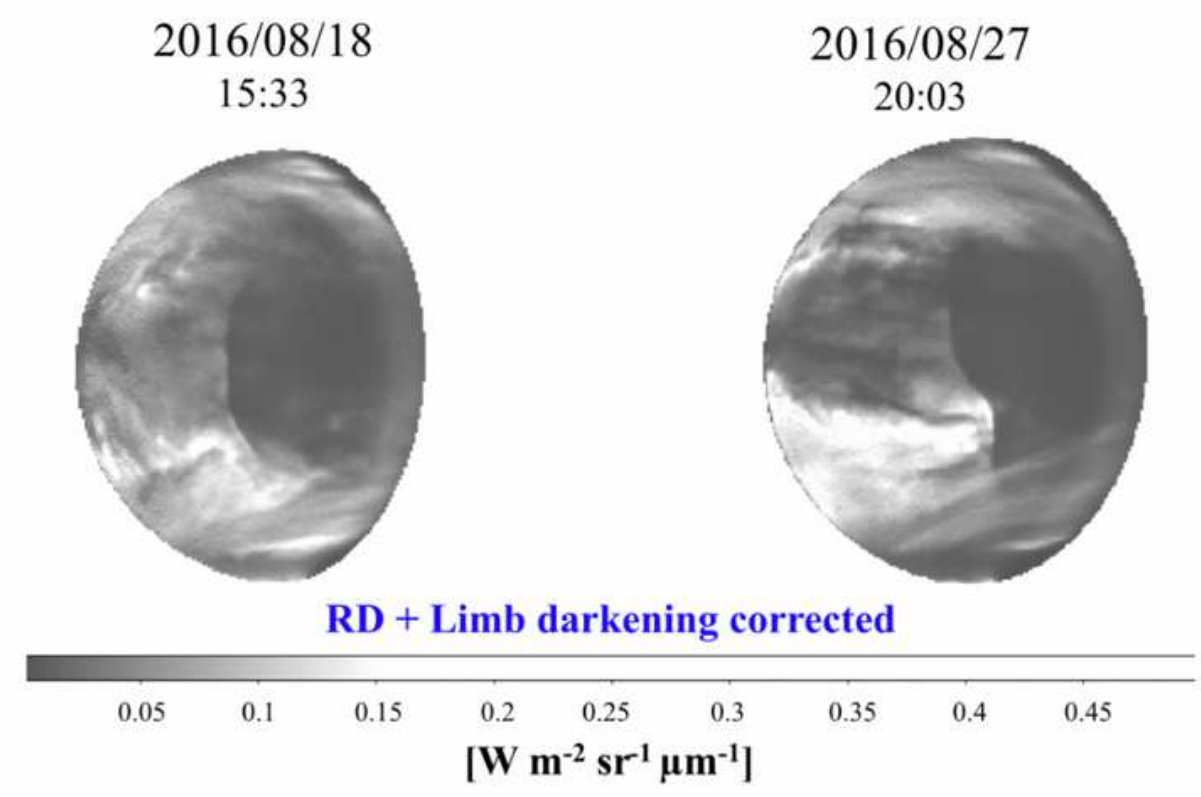

(b) RDD: $1.735 \mu \mathrm{m}$

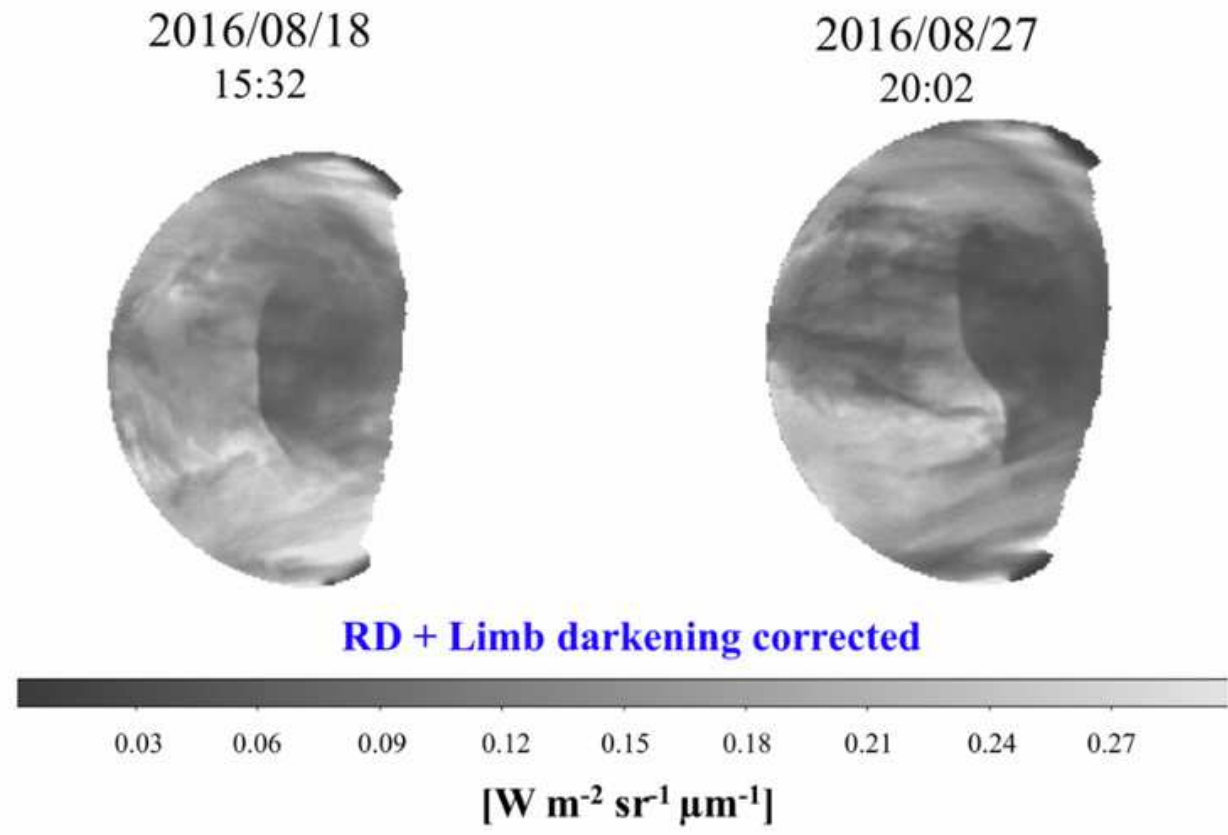

Figure 5

RDD image example in $2.26 \mu \mathrm{m}$ where the measurements for radiance contrast will be illustrated in Figure 8: (a) $2.26 \mu \mathrm{m}$; (b) $1.735 \mu \mathrm{m}$ 


\section{(a) 2016-08-18}

\section{$15: 33$}

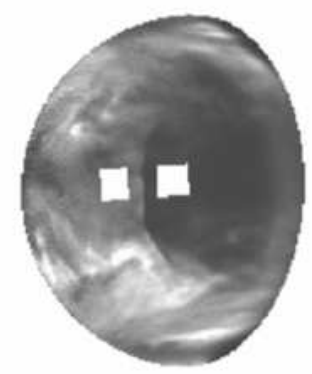

$17: 33$
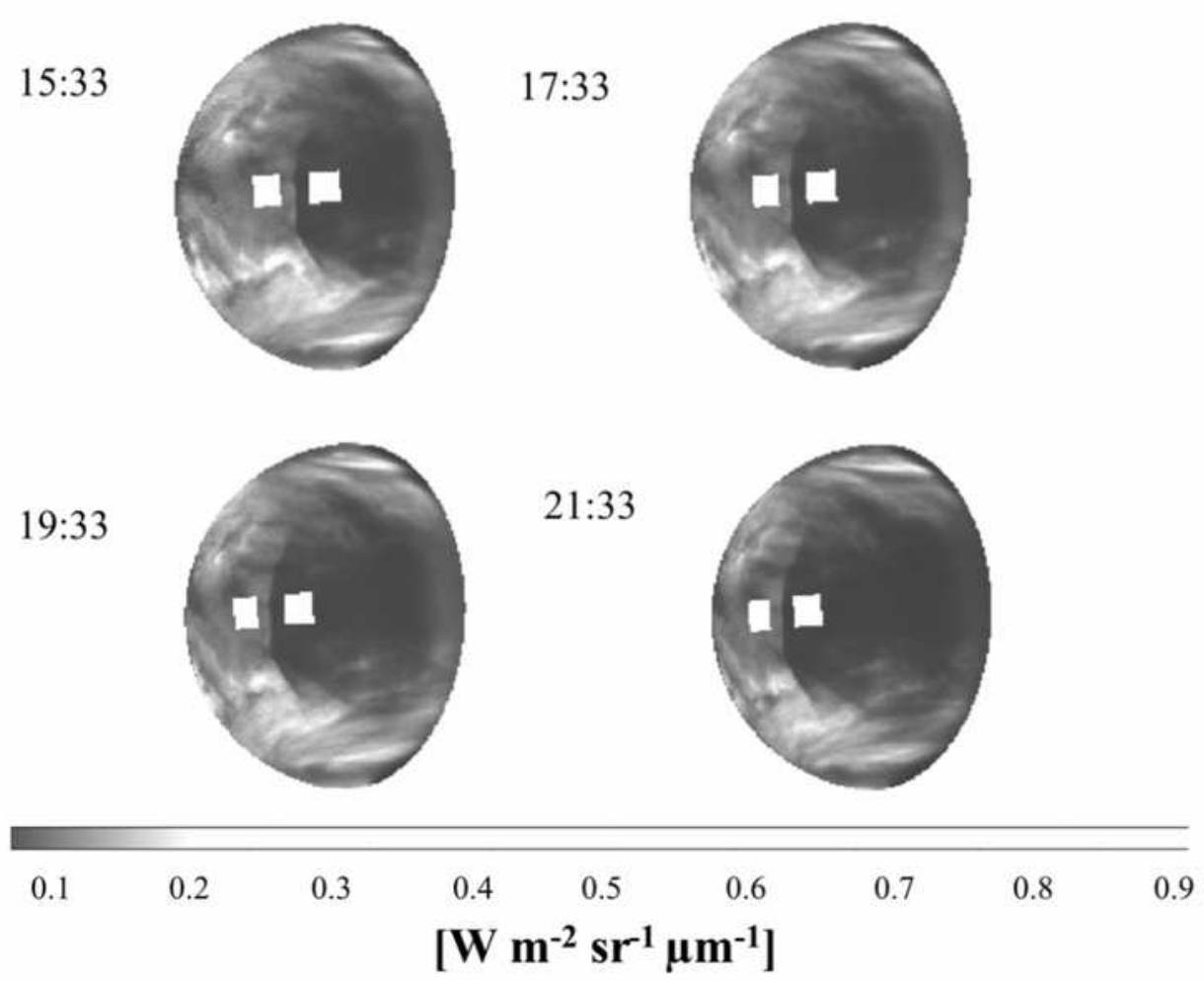

(b) 2016-08-27
22:03

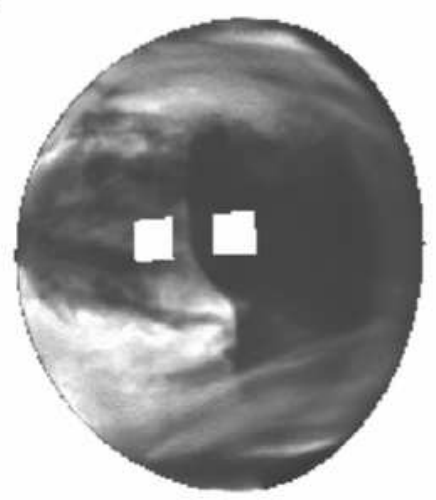

0.7

0.8

0.9

[W $\mathbf{~ m}^{-2} \mathbf{s r}^{-1} \mu \mathrm{m}^{-1}$ ]

\section{Figure 6}

$10^{\circ}$ longitude $\times 10^{\circ}$ latitude pixel 2 size 'box' to obtain average radiance within the selected region both in the optically thick enormous cloud and bright background ( $10^{\circ}$ longitude apart) to measure the averaged transmittance of radiation through the enormous cloud feature. The boxes are shifted longitudinally by estimated speed in between the 2-hr observations. Image examples shown here are 2-hr intervals of limb 
darkening corrected RD-restored data where the wind speed of the enormous cloud is estimated at 90 $\mathrm{m} / \mathrm{s}$. (a) $08-18 / 15: 33$ to $21: 33$; (b) $08-27 / 20: 03$ to $22: 03$

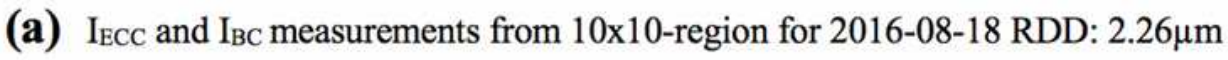

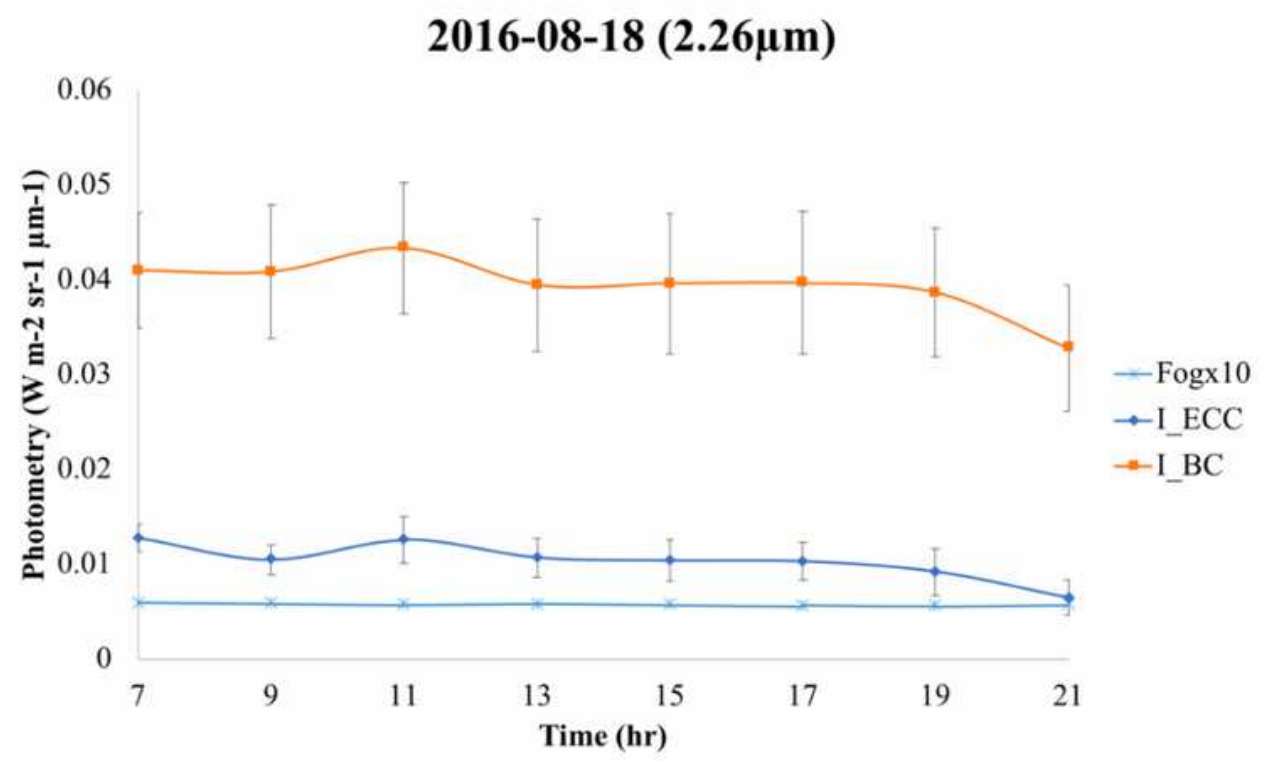

(b) $\mathrm{IECC}_{\mathrm{EC}}$ and $\mathrm{I}_{\mathrm{BC}}$ measurements from 10x10-region for 2016-08-18 RDD: 2.26 $\mu \mathrm{m}$

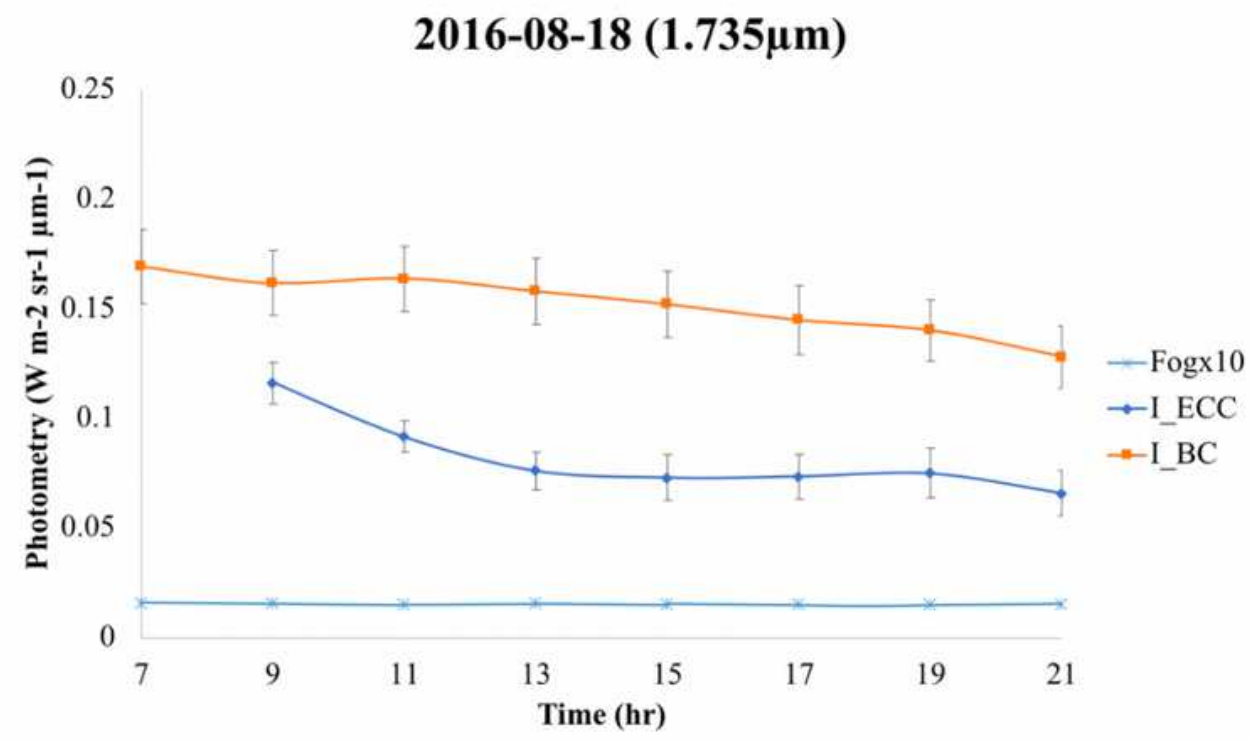

Figure 7

Average radiances of 'box region' in ECC and BC as selected in Figure 6a obtained for RDD images.

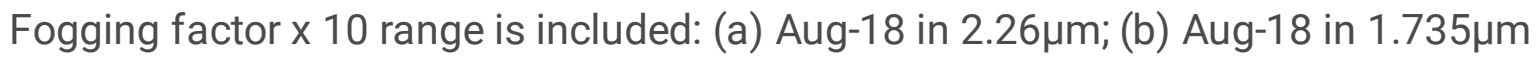


(a) $\mathrm{I}_{\mathrm{ECC}}$ and $\mathrm{I}_{\mathrm{BC}}$ measurements from 10x10-region for 2016-08-27 RDD: $2.26 \mu \mathrm{m}$

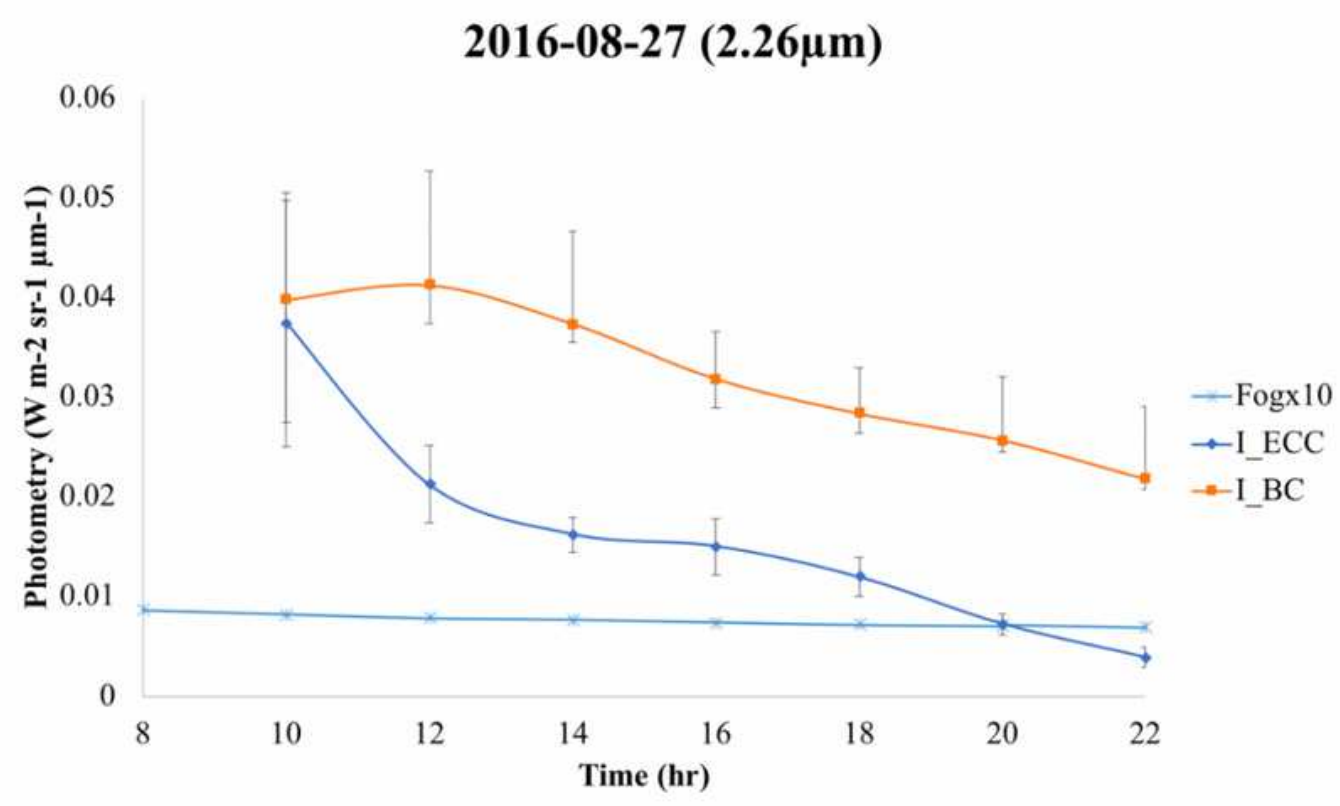

(b) $\mathrm{I}_{\mathrm{ECC}}$ and $\mathrm{I}_{\mathrm{BC}}$ measurements from 10x10-region for 2016-08-27 RDD: $2.26 \mu \mathrm{m}$

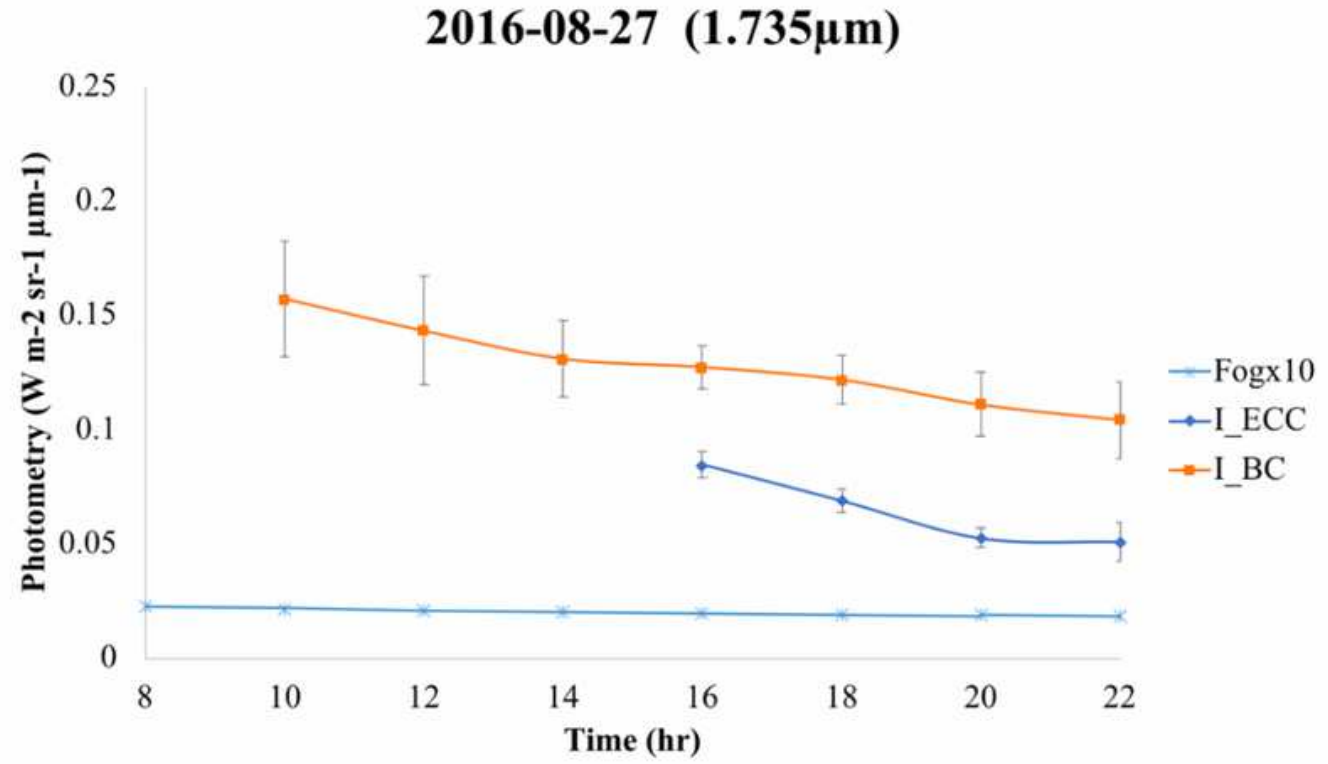

Figure 8

Average radiances of 'box region' in ECC and BC as selected in Figure 6b obtained for RDD images. Fogging factor $x 10$ range is included: (a) Aug-27 in 2.26 $1 \mathrm{~m}$; (b) Aug-27 in 1.735 $\mu \mathrm{m}$ 


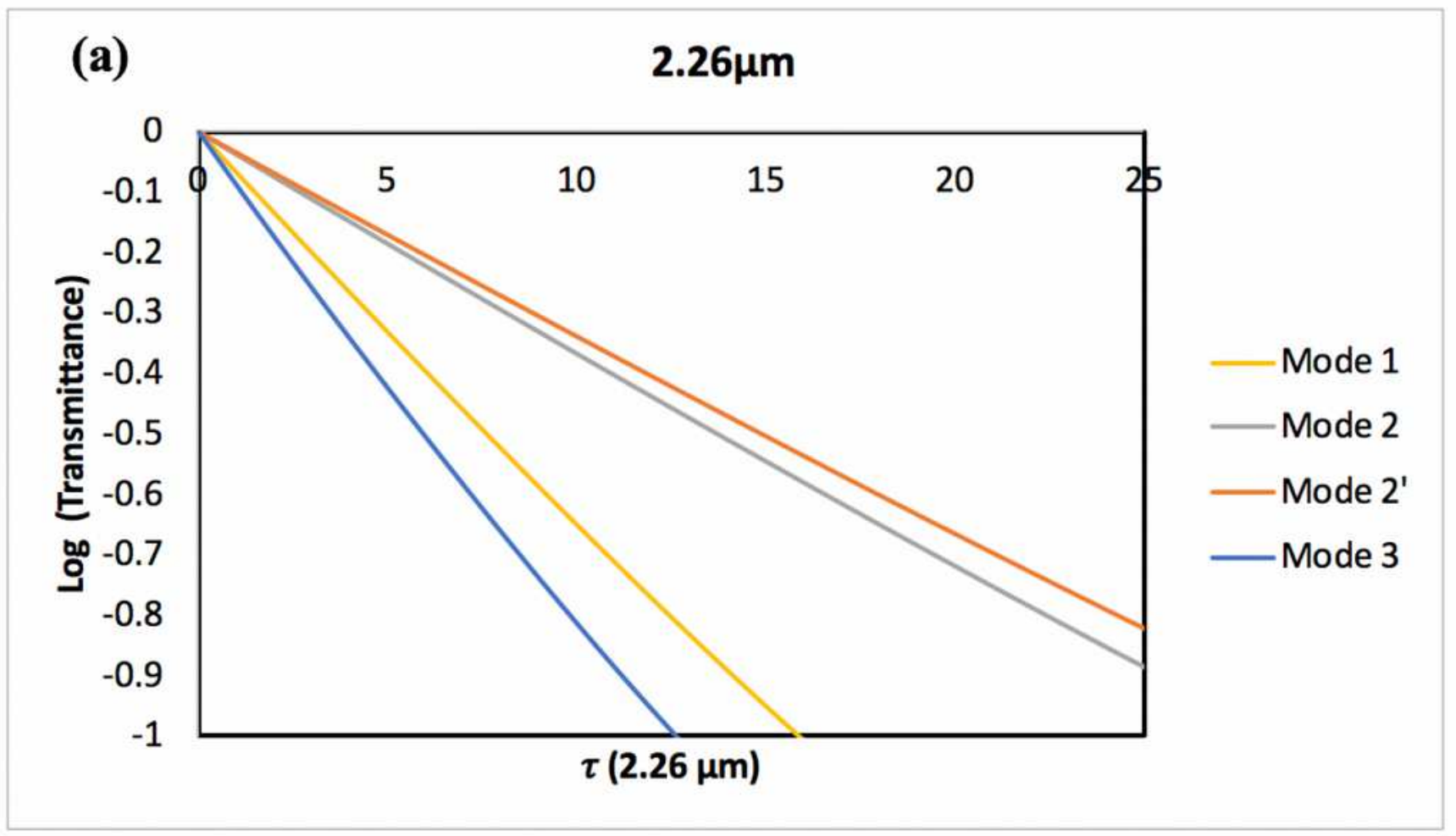

(b)

$1.735 \mu \mathrm{m}$

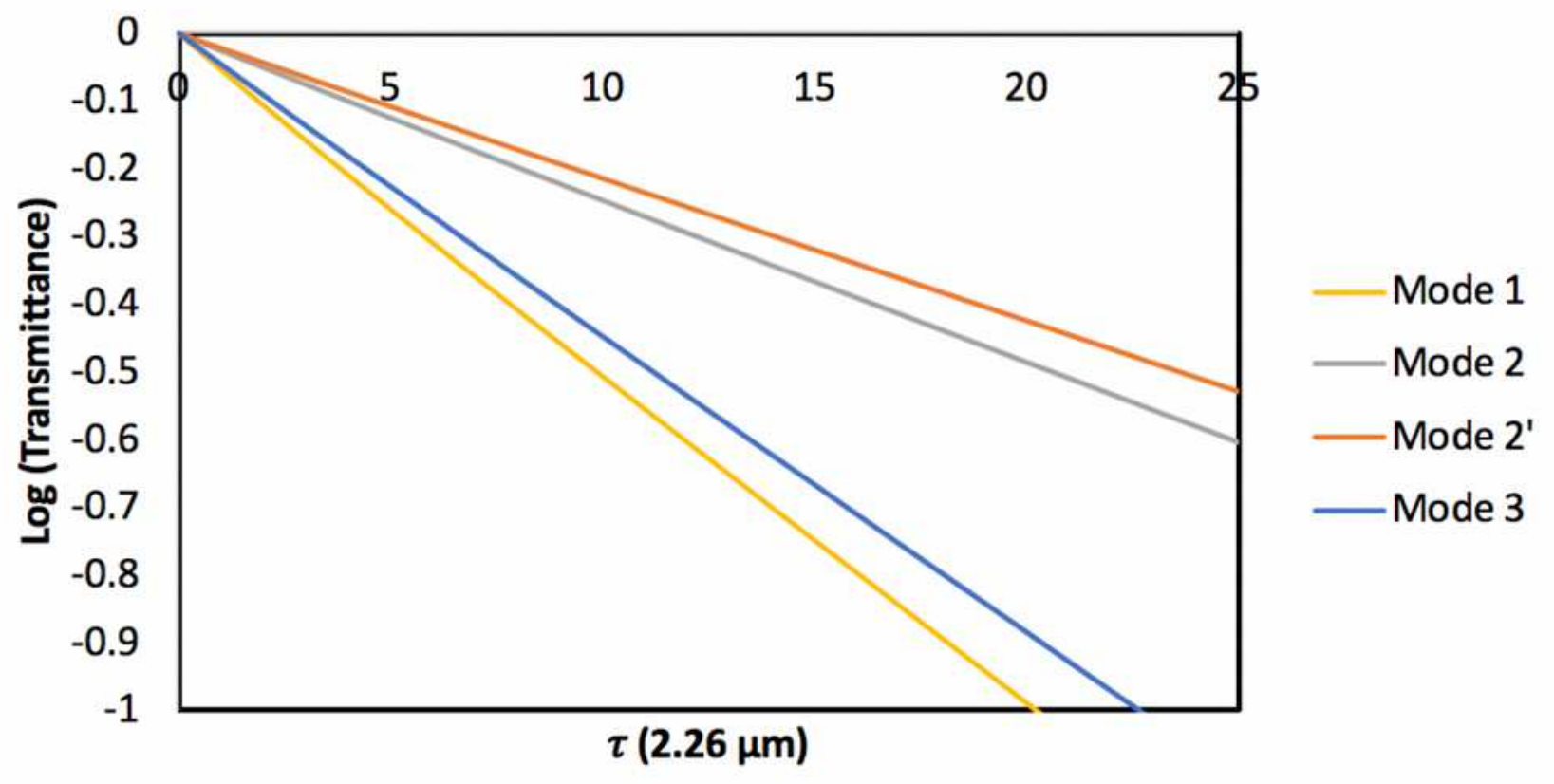

Figure 9

Radiative transfer calculation for transmittance by additional optical thickness of single-mode aerosols (mode 1, mode 2, mode 2', and mode 3) between 54 to $56 \mathrm{~km}$ altitude in: (a) $2.26 \mu \mathrm{m}$; (b) $1.735 \mu \mathrm{m}$. 


\section{Observed TR over theoretical TR}

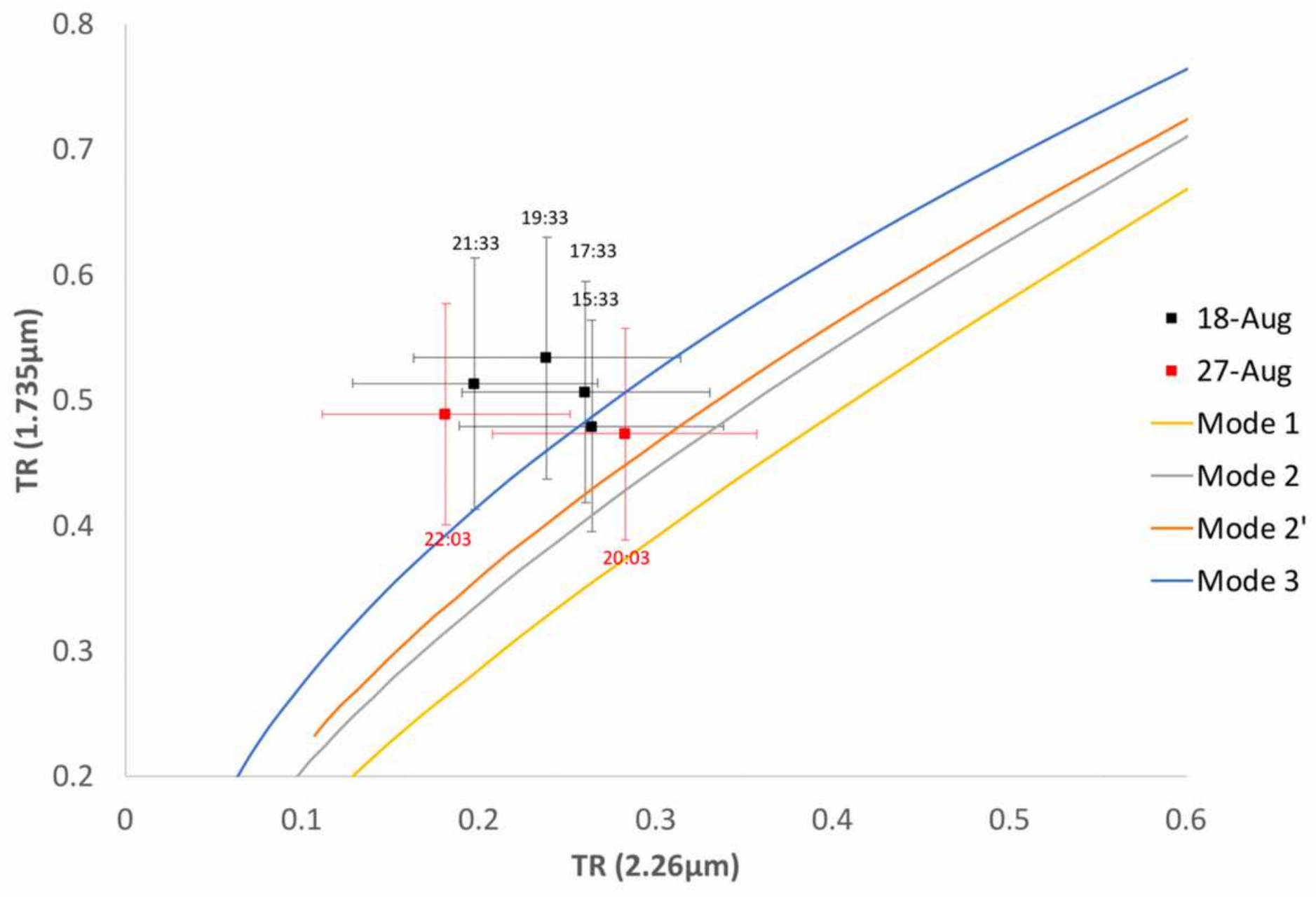

Figure 10

Observed Transmittance (TR) with associated uncertainty range plotting over theoretical Transmittance (TR) computed by radiative transfer for different particle size modes (mode 1, mode 2, mode 2', mode 3). 

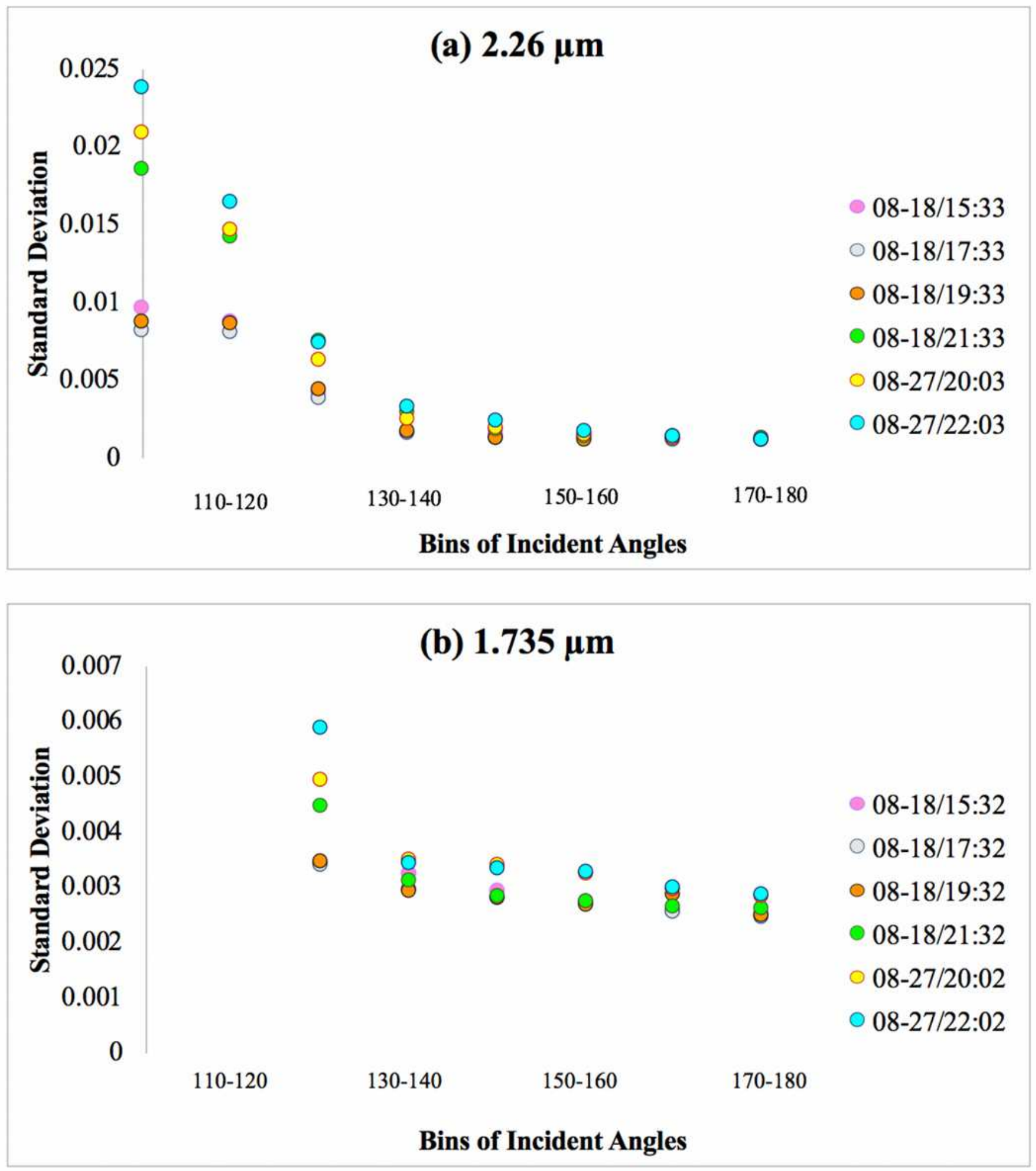

Figure 11

Standard deviation of CRD to L2B images in bins of $10^{\circ}$ incident angles for: (a) $2.26 \mu \mathrm{m}$; (b) $1.735 \mu \mathrm{m}$. There are no data for incident angles before $120^{\circ}$ due to saturation. 


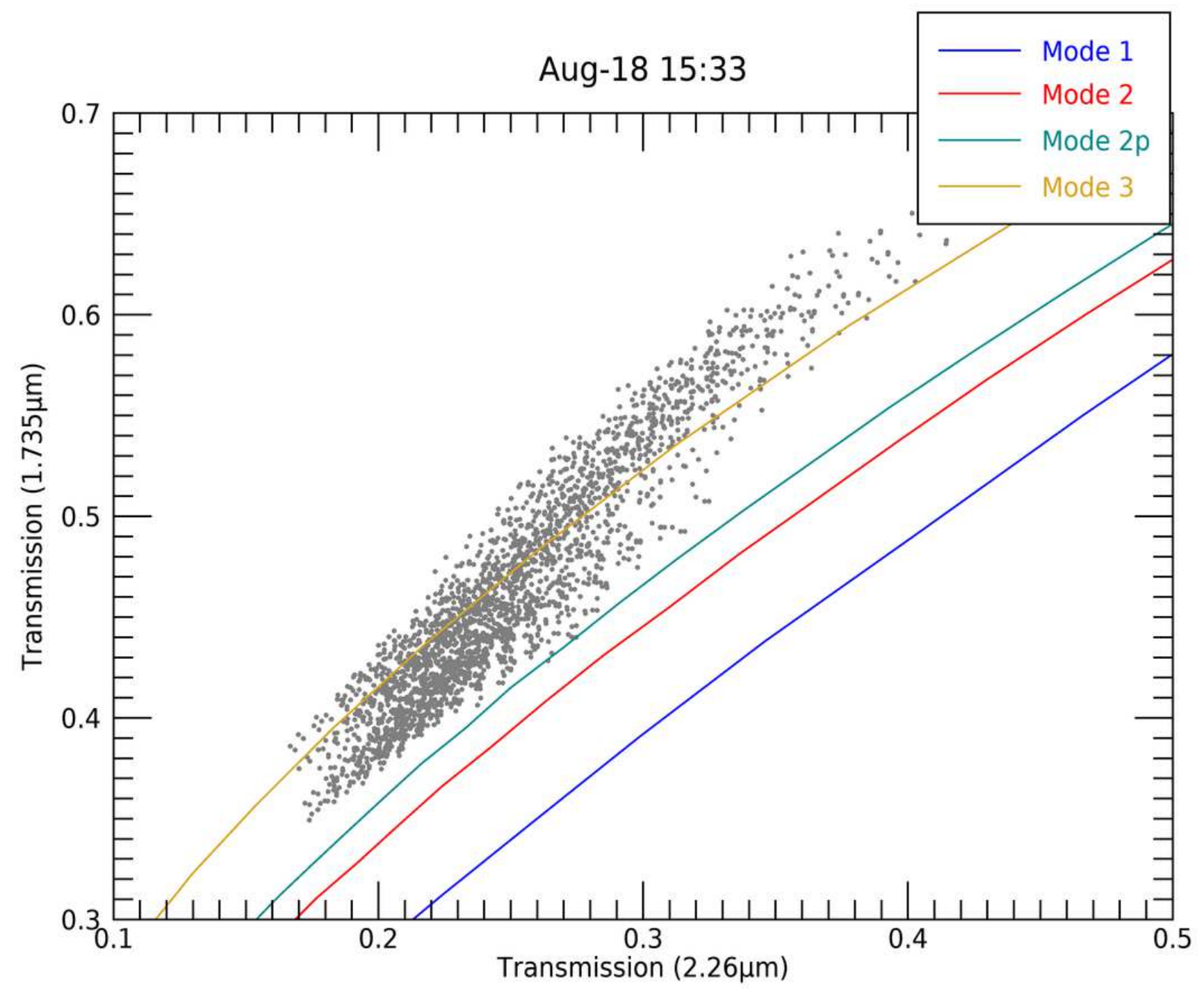

Figure 12

Total combinations of $4 \times 4$-regions' transmission ratios plotted over particle mode theoretical curves (mode 1, mode 2, mode 2' mode 3 ) showing alignment around mode 3 and slightly reaching mode 2' theoretical curves. 

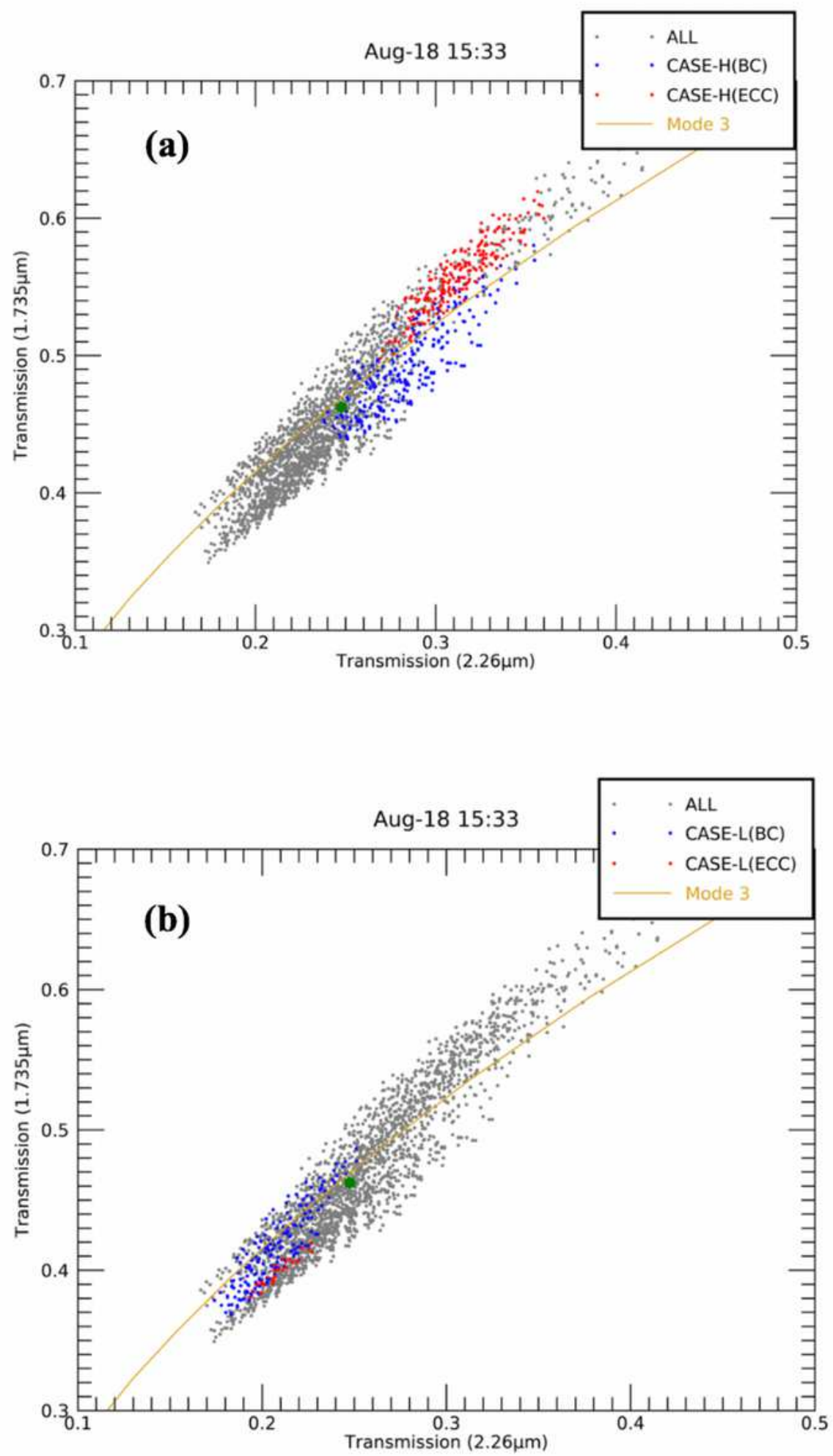

Figure 13

(a) Case-L in which to lower the transmissions. This is divided into two sub-groups. 'Case-L(BC)' where I_BC is brighter than 'normal' intensity and 'Case L(ECC)' where I_ECC is darker than normal intensity; (b) Case-H in which to increase to higher transmissions. This is divided into two sub-groups. 'Case-H(BC)' where I_BC is darker than 'normal' intensity and 'Case L(ECC)' where I_ECC is brighter than normal intensity 


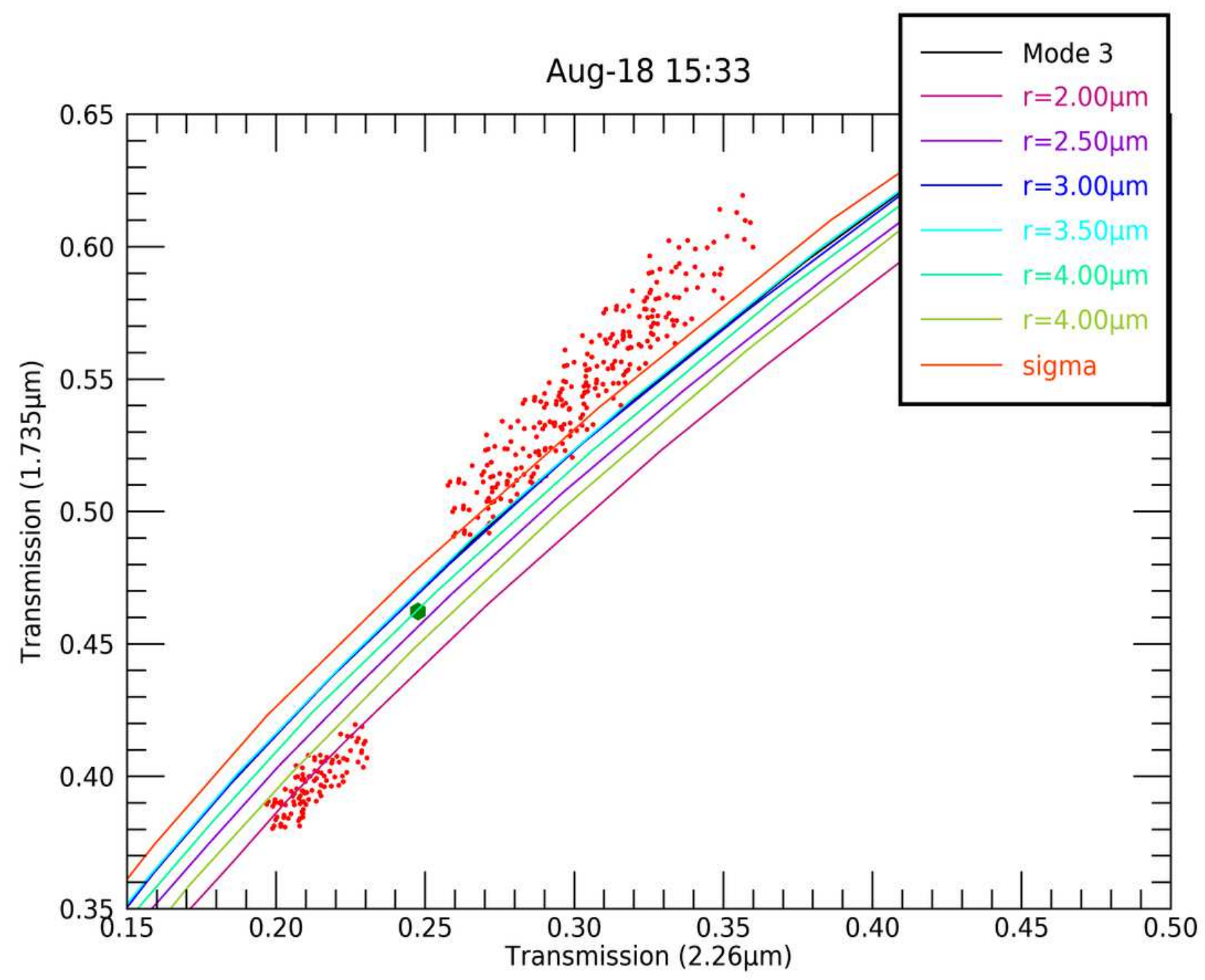

Figure 14

Theoretical transmittance calculated for mode $3^{\prime}$ ranging from $r \otimes=2.0 \mu \mathrm{m}$ to $4.5 \mu \mathrm{m}$ in $0.25 \mu \mathrm{m}$ intervals, and one fifth width of size distribution of mode 3 to the original denoted as ' $3.65 \mu \mathrm{m} \_s i g$ ', and mode 3 is $3.65 \mu \mathrm{m}$ mean radius. (Red Dots) are from previous Figure 13a and 13b representing I_ECC points. 


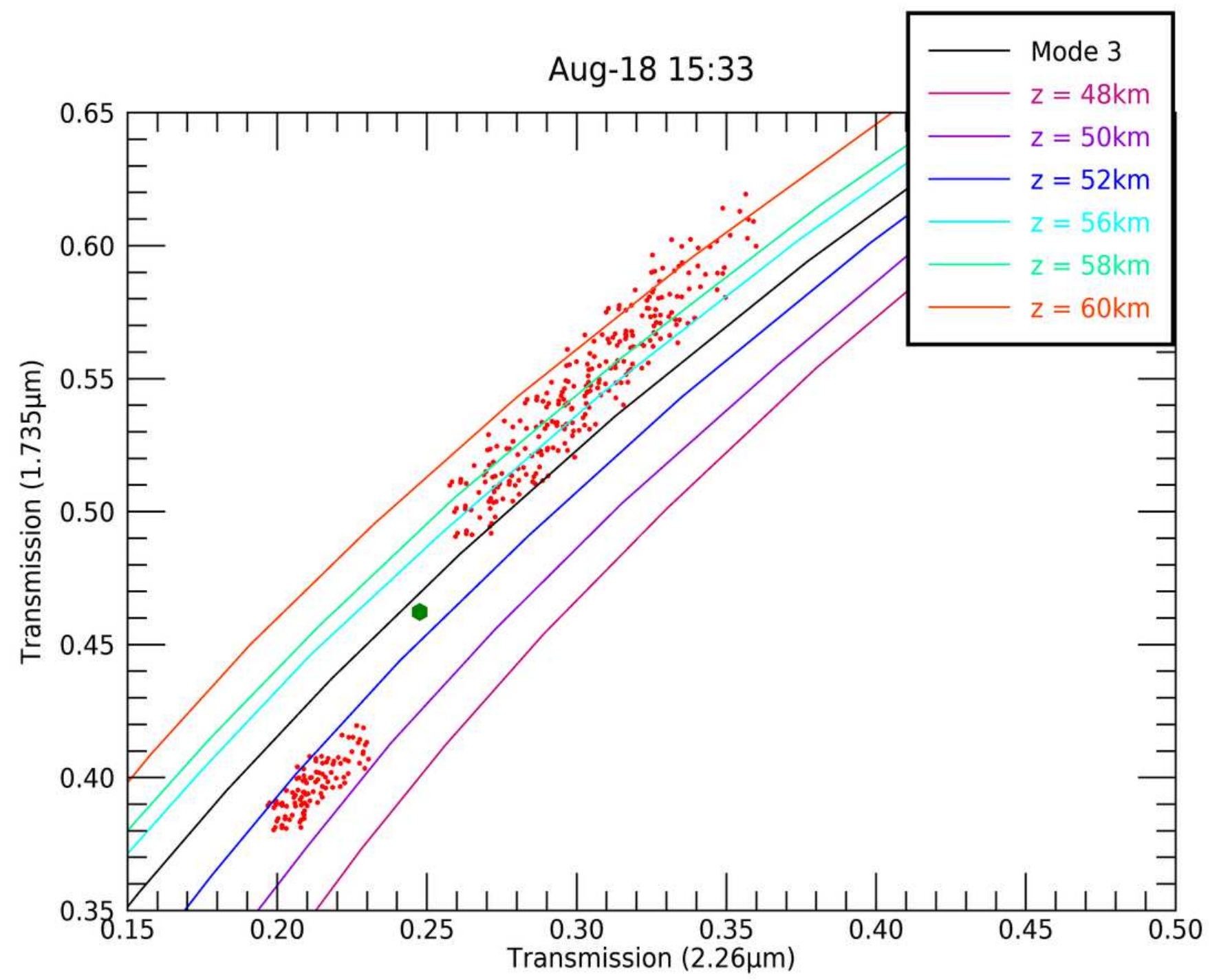

\section{Figure 15}

Radiative transfer calculations for emissions by single-mode aerosols (only mode 3 for this study) at different altitudes varying from $48-60 \mathrm{~km}$ (at $2 \mathrm{~km}$ intervals).
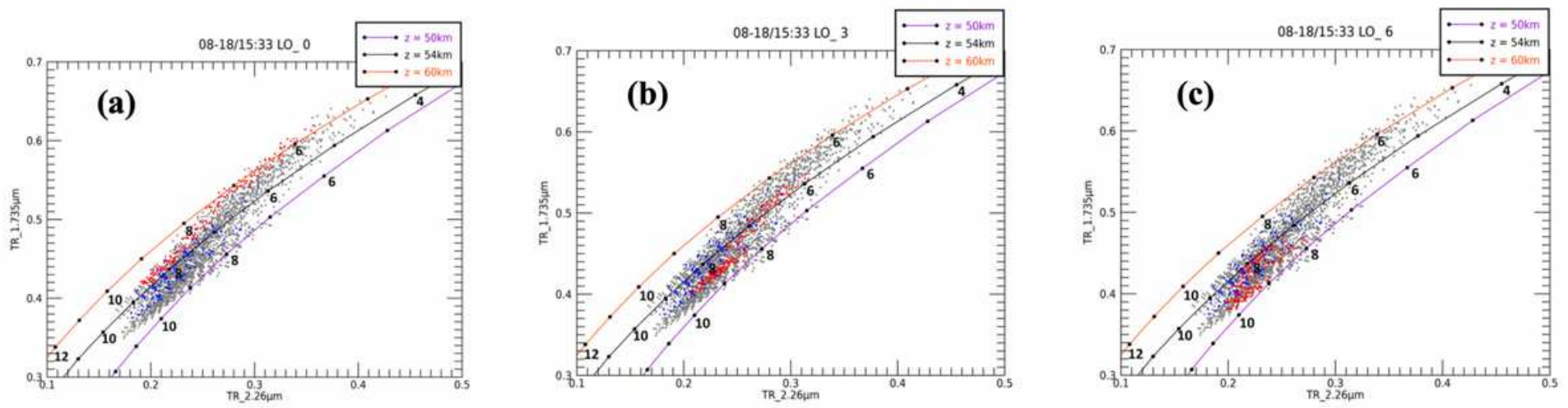

Figure 16 
Combination scatter plots within 10x10region with resolution of $4 \times 4$-region statistical analysis. (Red) Transmission distribution of the Enormous Cloud Cover 'ECC'. (Blue) Transmission distribution of the Background Cloud 'BC' in 08-18/15:33: (a) LO_0, (b) LO_3, (c) LO_6

\section{8-18/15:33}

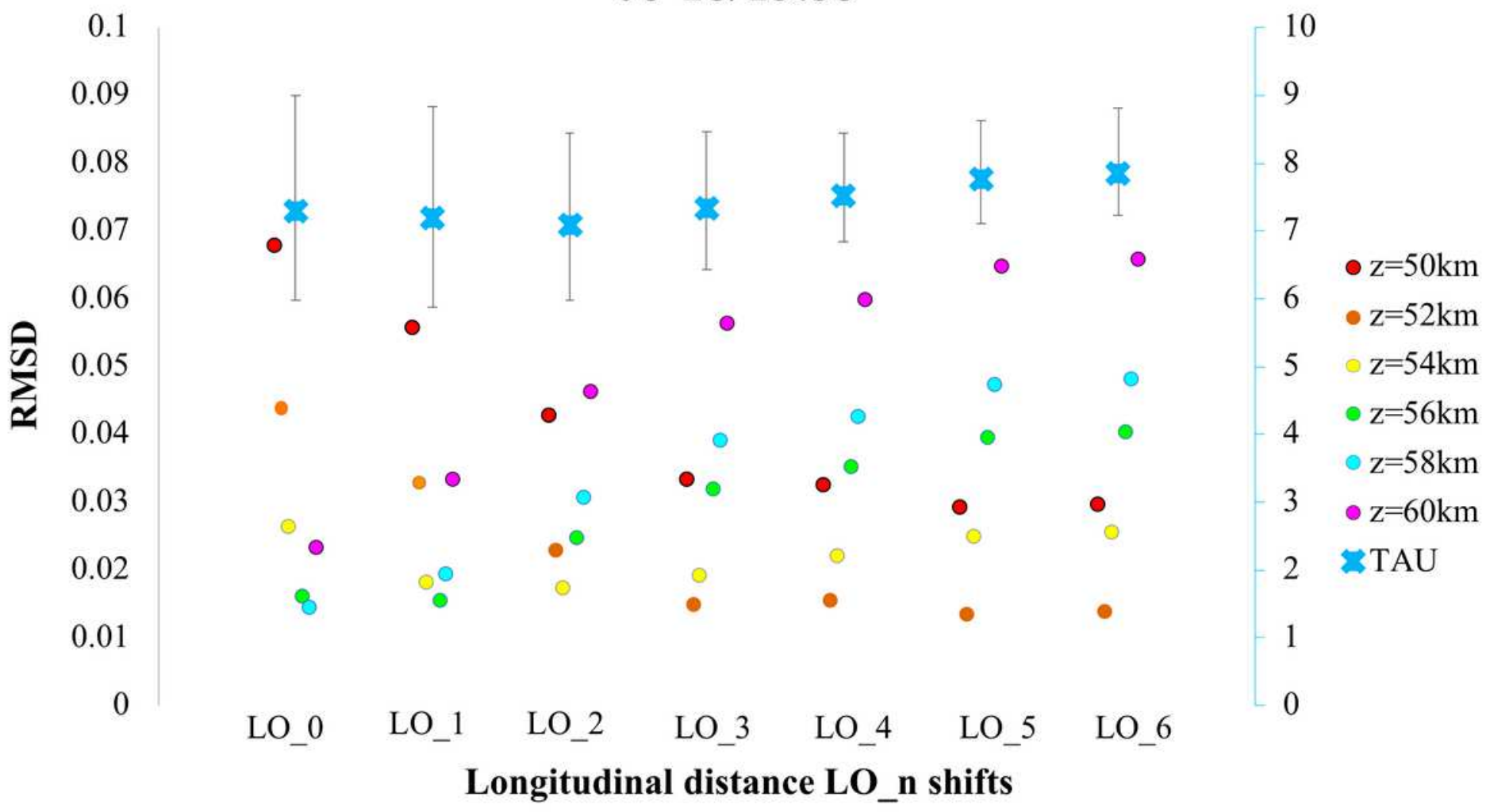

Figure 17

RMSD for longitudinal distance away from discontinuity front (spatial variation): 08-18/15:33. Left y-axis is RMSD and Right y-axis optical depths. 

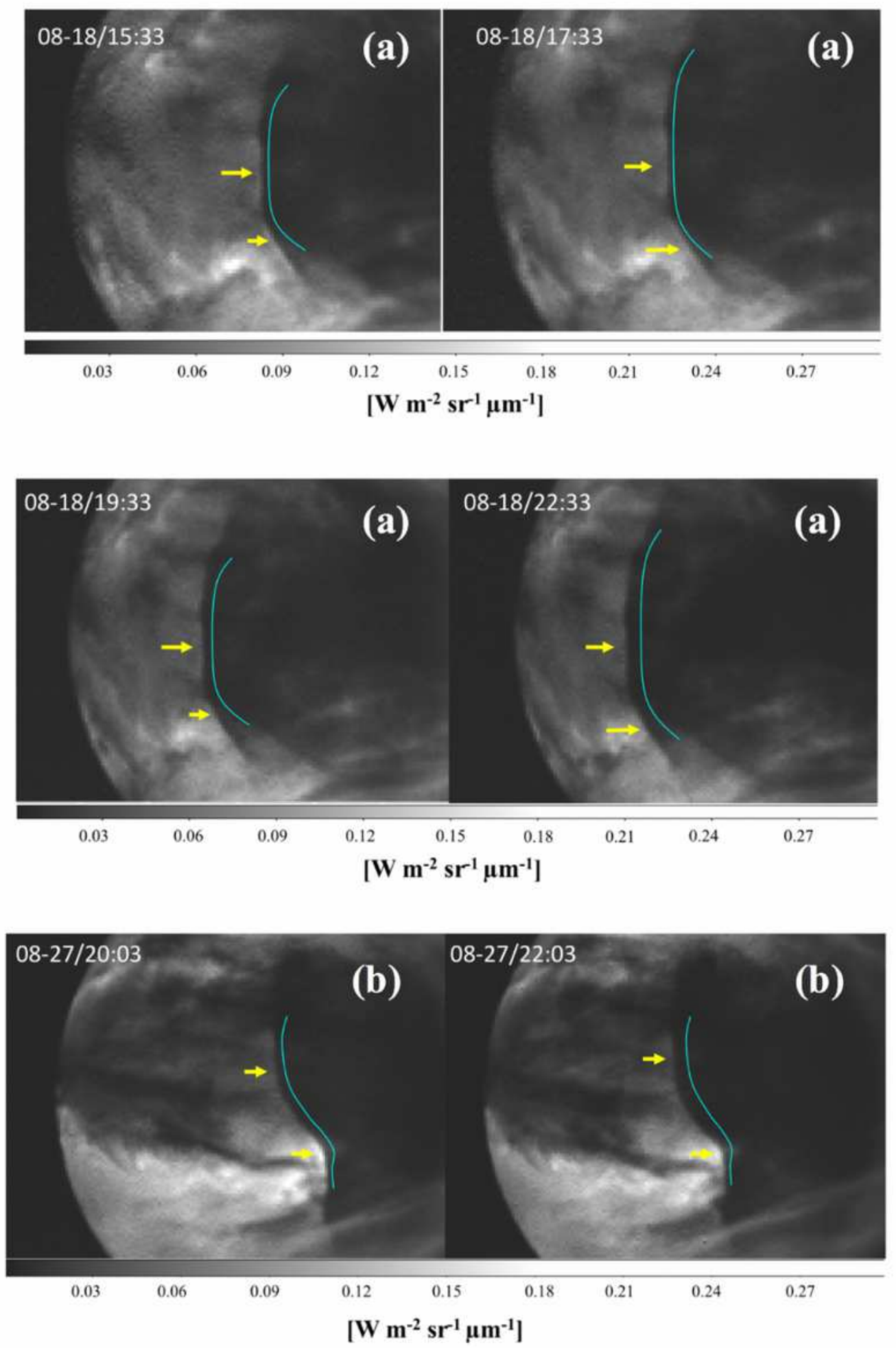

Figure 18

Close up image of RD-data (blue curve line) discontinuity front, (yellow arrow) bright downwelling features. (a) for $08-18 / 15: 33,17: 33,19: 33$, and $21: 33$. Note on the bright strip just after the front is most prominent at 15:33, then decreasing gradually in radiance to 21:33; (b) 08-27/20:03 and 22:03. 


\section{Traveling Westward}

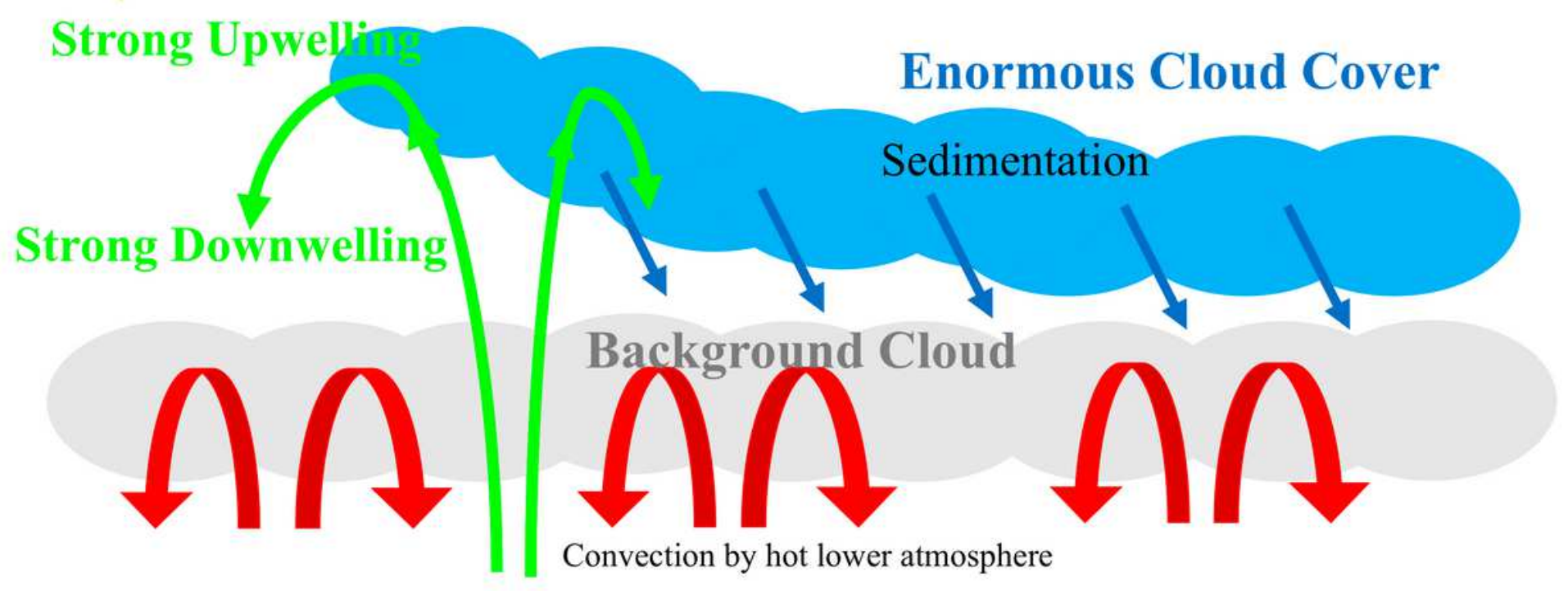

Figure 19

Schematics of ECC
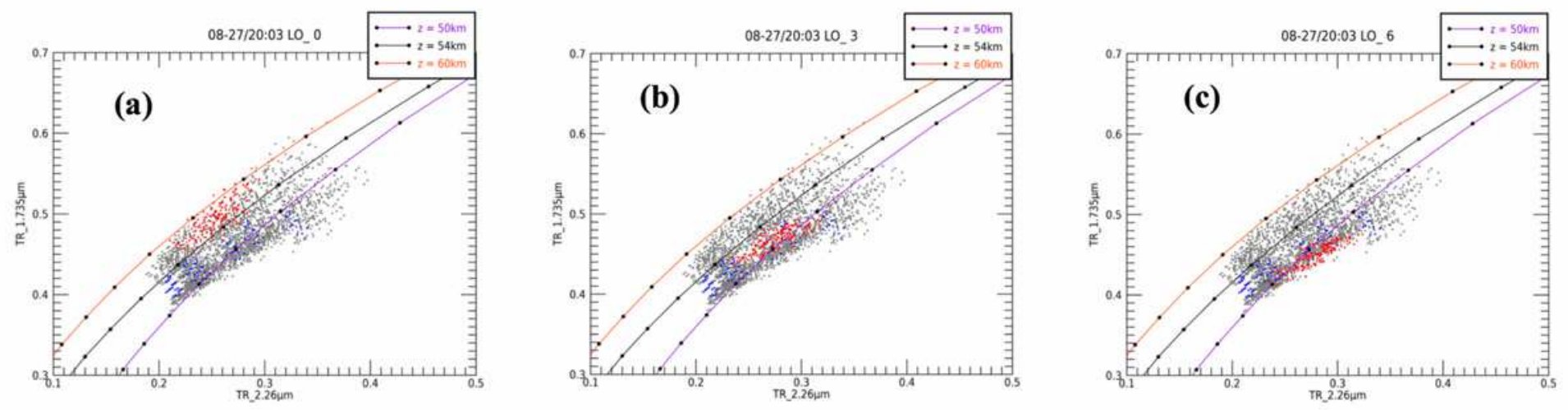

Figure 20

Combination scatter plots within 10x10region with resolution of 4x4-region statistical analysis. (Red) TR4 of the Enormous Cloud Cover 'ECC'. (Blue) Transmission distribution of the Background Cloud 'BC' in 0827/20:03: (a) LO_0, (b) LO_3, (c) LO_6 


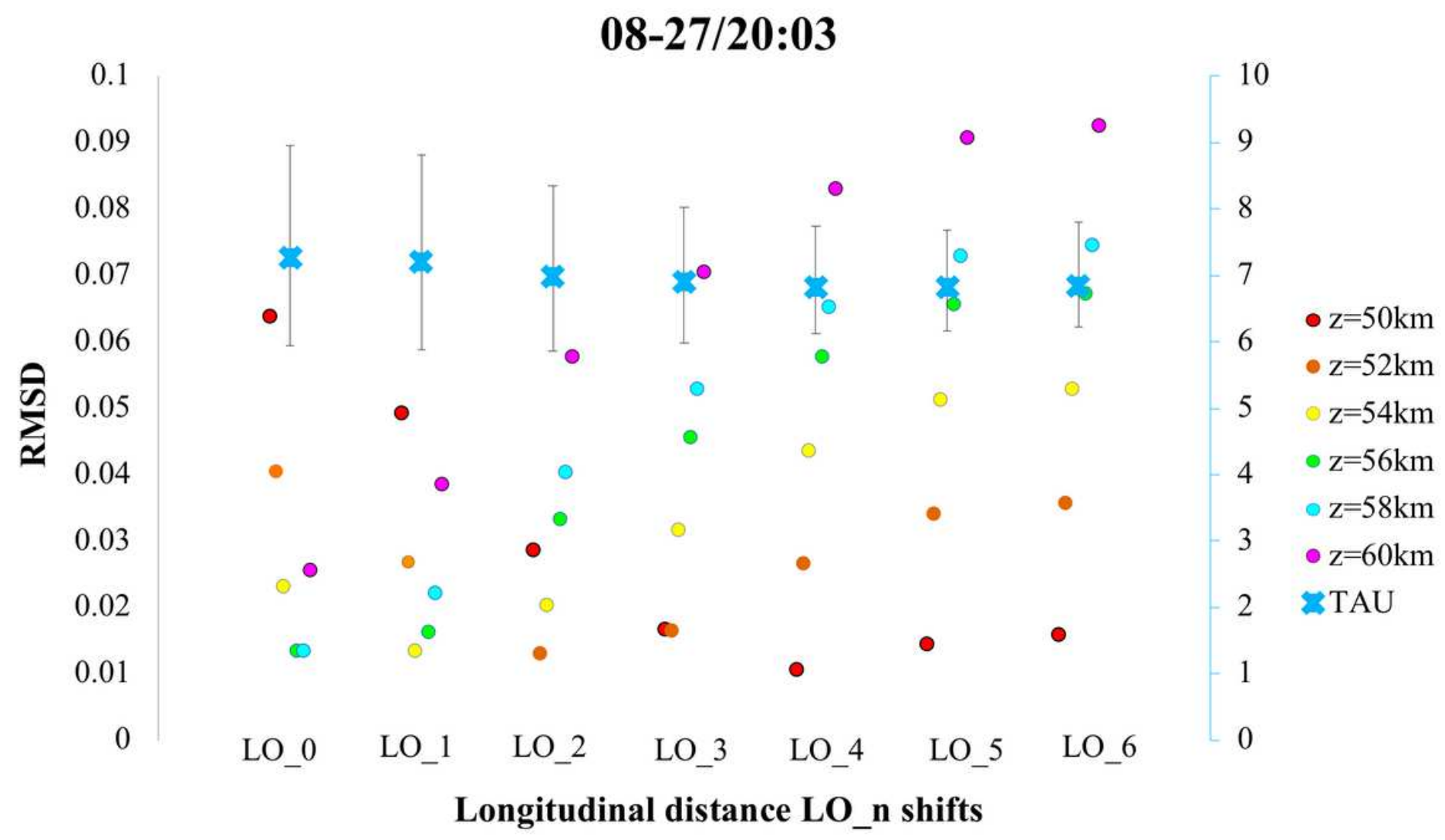

Figure 21

RMSD for longitudinal distance away from discontinuity front (spatial variation): 08-27/20:03. Left y-axis is RMSD and Right y-axis optical depths.

\section{Supplementary Files}

This is a list of supplementary files associated with this preprint. Click to download.

- TablesVun.docx 